www.mdpi.com/journal/pharmaceuticals

Review

\title{
The Chick Embryo Chorioallantoic Membrane as an In Vivo Assay to Study Antiangiogenesis
}

\section{Domenico Ribatti}

Department of Human Anatomy and Histology, University of Bari Medical School, Piazza G. Cesare, 11, Policlinico 70124, Bari, Italy; E-Mail: ribatti@anatomia.uniba.it; Tel.: +39-080-547-8240; Fax: $+39-080-547-8310$.

Received: 12 January 2010; in revised form: 29 January 2010 / Accepted: 2 March 2010 / Published: 8 March 2010

\begin{abstract}
Antiangiogenesis, e.g., inhibition of blood vessel growth, is being investigated as a way to prevent the growth of tumors and other angiogenesis-dependent diseases. Pharmacological inhibition interferes with the angiogenic cascade or the immature neovasculature with synthetic or semi-synthetic substances, endogenous inhibitors or biological antagonists. The chick embryo chorioallantoic membrane (CAM) is an extraembryonic membrane, which serves as a gas exchange surface and its function is supported by a dense capillary network. Because its extensive vascularization and easy accessibility, CAM has been used to study morphofunctional aspects of the angiogenesis process in vivo and to study the efficacy and mechanism of action of pro- and anti-angiogenic molecules. The fields of application of CAM in the study of antiangiogenesis, including our personal experience, are illustrated in this review article.
\end{abstract}

Keywords: antiangiogenesis; chorioallantoic membrane; tumor progression

\section{Introduction}

Angiogenesis depends on the balance of several stimulating and inhibiting factors [1]. Antiangiogenesis as a way of treating primary tumors and reducing their metastases, was first proposed by Judah Folkman in 1971 [2]. Beginning in the 1980s, the biopharmaceutical industry began exploiting the field of antiangiogenesis for creating new therapeutic compounds for modulating new blood vessel growth in angiogenesis-dependent diseases. 
Antiangiogenic approaches fell in two categories: (a) agents that blocked the activity of pro-angiogenic molecules; (b) agents that directly affected endothelial cell function or survival (Table 1).

Table 1. Direct and indirect angiogenesis inhibitors.

\begin{tabular}{|l|l|}
\hline \multicolumn{1}{|c|}{ Direct } & \multicolumn{1}{c|}{ Indirect } \\
\hline Angiostatin; Bevacizumab (Avastin) & Targeting EGF-receptor tyrosine kinase \\
Arresten; Canstatin; Combrestatin & Targeting VEGF receptor \\
Endostatin; Thrombospondin & Targeting PDGF receptor \\
Tumstatin; methoxyestradiol; Vitaxin & Targeting PDGF receptor \\
& Targeting ERBB-2 \\
& Targeting interferon alpha receptor* \\
\hline
\end{tabular}

* Interferon alpha can be considered both a direct angiogenesis inhibitor, because it inhibits endothelial-cell migration, and an indirect angiogenesis inhibitor because it inhibits synthesis of FGF-2 by tumor cells.

Endogenous inhibitors of angiogenesis are defined as proteins or fragments of proteins that are formed in the body and can inhibit the formation of blood vessels (Table 2) [3].

Table 2. Endogenous inhibitors of angiogenesis.

\begin{tabular}{|c|c|}
\hline Matrix derived & Growth factors and cytokines \\
\hline $\begin{array}{l}\text { Arresten } \\
\text { Canstatin } \\
\text { Endorepellin } \\
\text { Endostatin } \\
\text { Fibronectin fragment (Anastellin) } \\
\text { Targeting fibronectin-binding integrins } \\
\text { Fibulin } \\
\text { Thrombospondin-1 and }-2 \\
\text { Tumstatin }\end{array}$ & $\begin{array}{l}\text { Interferons } \\
\text { Interleukins } \\
\text { Pigment epithelium derived factor (PEDF) }\end{array}$ \\
\hline Fragments of blood coagulation factors & Others \\
\hline $\begin{array}{l}\text { Angiostatin } \\
\text { Antithrombin-III } \\
\text { Prothrombin kringle } 2 \\
\text { Platelet factor-4 }\end{array}$ & $\begin{array}{l}\text { Tissue inhibitors of metalloproteinases (TIMPs) } \\
\text { Chondromodulin } \\
\text { 2-methoxyestradiol } \\
\text { Prolactin fragments } \\
\text { PEX } \\
\text { Soluble Fms-like tyrosine kinase-1 (S-Flt-1) } \\
\text { Troponin I } \\
\text { Vasostatin }\end{array}$ \\
\hline
\end{tabular}

At least 27 different proteins and small molecules exist in the body whose function is to act as inhibitors of angiogenesis. These angiogenesis inhibitors can be detected in blood circulation, suggesting that they function in the angiogenic balance as endogenous angiostatic regulators even under physiological conditions [3]. 
Today, over 20 angiogenic growth factors and over 300 antiangiogenic molecules targeting different signalling pathways are being tested for their anticancer properties at preclinical and clinical stages. Although the results of the clinical trials are encouraging, the effects are modest. Clinical practice reveals that therapy with angiogenesis inhibitors does not prolong survival of cancer patients for more than months, because tumors elicit resistance.

Owing to its central role in promoting tumor growth, vascular endothelial growth factor (VEGF) has become a key therapeutic target and its function can be blocked at different levels of the signalling pathways. The majority of Food and Drug Adminstration (FDA)-approved angiogenesis inhibitors, as well as those in phase III clinical trials, neutralize VEGF, target its receptors or suppress its expression by tumor cells. Avastin (bevacizumab), a humanized anti-VEGF monoclonal antibody, has been the first angiogenesis inhibitor that was tested in multi-center clinical trials against cancer. In 2004, it was approved by the FDA as a first-line treatment for metastatic clorectal cancer in combination with 5-fluorouracil-based chemotherapy regimens [4].

\section{The Chorioallantoic Membrane in the Study of Antiangiogenesis}

The classical assays for studying angiogenesis in vivo include the rabbit ear chamber, the mouse dorsal skin and air sac, the chick embryo chorioallantoic membrane (CAM), the iris and avascular cornea of the rodent eye and the zebrafish [5].

In vivo angiogenesis assays have allowed important progress in elucidating the mechanism of action of several angiogenic factors and inhibitors. The main determinants dictating the choice of method are their cost, ease of use, reproducibility, and reliability. However, in vivo angiogenesis assays may be very sensitive to environmental factors and not readily accessible to biochemical analysis. Also, their interpretation is frequently complicated by the fact that the experimental condition adopted may inadvertently favour inflammation. In this case the angiogenic response is elicited indirectly, at least in part, through the activation of inflammatory or other non-endothelial cell types.

The CAM is an extraembryonic membrane formed on day 4 of incubation by fusion of the chorion and the allantois. Immature blood vessels, lacking a complete basal lamina and smooth muscle cells, scattered in the mesoderm grow very rapidly until day 8 and give rise to a capillary plexus, which comes to be intimately associated with the overlying chorionic epithelial cells and mediates gas exchange with the outer environment. At day 14, the capillary plexus is located at the surface of the ectoderm adjacent to the shell membrane. Rapid capillary proliferation continues until day 11; thereafter, the endothelial cell mitotic index declines rapidly, and the vascular system attains its final arrangement on day 18, just before hatching [6].

CAMs are cultured either in ovo or ex-ovo as a shell-less culture in Petri dishes and plastic wrap/cup apparatus. There is no clear evidence that there is any significant difference between data derived using in ovo or shell-less culture method. It has been demonstrated that survival rate of eggs cultured ex ovo is the major success limiting step in this culture technique [6].

Focal application of test and control substances is still the most used method. It is quick and semi-quantifiable, economical, good for the screening of many novel substances. The one limitation of this approach concerns quantification of interaction of antiangiogenic drugs with CAM vessels rather than with pro-angiogenic molecules [6]. 
There are a variety of application methods or carriers described in literature to test angiogenic or antiangiogenic activity. The test material is usually introduced in the form of small filter disks, or small pieces of polymerized materials, such as gelatin sponges or biologically inert synthetic polymers. Blood vessels can be analyzed in terms of the number, diameter, density, permeability, branch point number and blood flow [6].

We have developed a new method for the quantitation of angiogenesis and antiangiogenesis in the CAM. Gelatin sponges treated with a stimulator or an inhibitor of blood vessel formation are implanted on growing CAM on day 8 [7]. Blood vessels growing vertically into the sponge and at the boundary between sponge and surrounding mesenchyme, are counted morphometrically on day 12 . The newly formed blood vessels grow perpendicularly to the plane of the CAM inside the sponge, which does not contain preexisting vessels and can be quantified by morphometric evaluation of histologic CAM sections. More sophisticated techniques have been designed recently to perform reliable quantitative evaluation of vascular density, including in ovo cell proliferation, layered expression scanning to visualize the protein of interest, and fluorescent confocal microscopy of new blood vessels formation at the site of application.

The development of an avascular zone or a zone of inhibition at the site of application is considered indicative of antiangiogenesis. It was initially described by Taylor and Folkman [8] who showed that protamine produced an avascular zone when applied to the leading edge of the CAM. In studies of inhibition of angiogenesis (Table 3), there are two approaches which differs in the target vessels, i.e. those which examine the response in the rapid growing CAM blood vessels and those that evaluate the inhibition of angiogenic response induced by a well known angiogenic cytokine, usually fibroblast growth factor-2 (FGF-2) or VEGF.

Table 3. Testing Antiangiogenic Substances in the CAM Assay.

AAV-mediated gene transfer of TIMP-1 [9]; AA98V (H)/L [10]; A-beta peptides [11]; Aeroplysinin-1[12]; Adiponectin [13]; Ad-vasostatin[14]; Agkistin [15]; AGM-1470 [16]; Alliin [17]; a4-b1antagonists[18]; Av-b3/av-b5 antagonists and ab [19,20,30,84,85,165,313]; Amifostine [21]; Amiloride [22]; Aminopeptidase-N antagonists [23]; Angioinhibins [24]; Ang-2 [25]; Angiostatin [26]; Angiotensinogen [27,28]; Anthracyclines and titanocene dichloride [29]; Antibacterial substances [31]; Antibiotics [32]; Ab anti-FGF-2 and anti-VEGF [33,34]; Anti-CD146 Mab [35]; Anti-collagen IV ab [36]; Antioxidant molecules [37]; Antithrombin [38]; Apicidin [39]; Aplidine [40]; Apolipoprotein(a) kringle V [41]; Apomorphine [42]; AQP-1 siRNA [43]; Arginine deaminase [44]; 2-aroylindoles [45]; Arresten [46]; Artesunate [47]; Ascorbic acid [48]; Atiprimod [49]; Aurintricarboxylic acid [50]; Azaspirine [51]

Bactericidal/permeability-increasing protein [52]; Bacterium PB[53]; Baicalein/baicalin [54]; Bleomycin [55]; Blockers of volume-regulated anion channels [56]; Beta-cyclodextrintetradecasulfate [57]; Beta-Escin [58]; Beta-HISV[59]; BMP-9 [60]; Bortezomib [61]; Butyric acid [62]

CAI [63]; Campesterol [64]; Canstatin [65]; Capsaicin [66]; Carbon materials [67]; Carrageenan [68]; Cartilage [69]; Catechins [70]; Cerivastatin [71]; Cheiradone [72]; Chemokine antagonist M3 [74]; Chondrocyte derived inhibitor [69]; Chondromodulin-1 [75]; Chrysin [76]; Cigarette smoke condensate [77,191]; Clodronate [78]; Clotrimazole [79]; Contortrostatin [80]; Curcumin [81,82]; COX inhibitors [86,87]; Cyclopeptidic VEGF inhibitor [88]; Cyclosporin [89]; Cytocholasin D [90]

7-Deazaxanthine [91]; Deguelin [92]; Delphinidin [93]; Deoxycholic acid-heparin conjugate [94]; Deoxycytidine nucleoside [95]; DFMO $\alpha$-difluoromethylornithine [96]; Diaminoanthraquinone [97]; Dichloropy ridodithienenotriazine [98]; Dihydroartemisinin [99]; Dihydrotanshinone I [100]; Digoxin [101]; Ditriazine derivative [102]; DPTH-N10 [103]; Docetaxel [104]; Dominant-negative p65 PAK peptide [105]; Doxazosin [106]; Doxycycline [107]; Doxorubicin [108,209] 
Table 3: Cont.

Eclipta prostata [109]; Emodin [110]; Endocannabinoid anandamide[111]; Endorepellin [112]; Endostatin [113,114,242]; Enoic acanthonic acid [86]; Eponeomycin [115]; Epoxyeicosatrienoic acid antagonist [116]; Escherichia Coli K5 polysaccharide derivatives [73,299]; Estrogen antagonists [117]; Ets-1 antisense [118,119]; Evodiamine [120]

Fascaplysin [121]; Fenretinide [122]; Flavonoids [123,261]; Fluorosynerazols [124]; Fractalkine [125]; Gangliosides [126]; Gastrodia elata [127]; Genepin [128]; Ghrelin [129] Gleditsia sinesis [130]; Glycine [131]; Goniodomin A [132] Grateloupia longifolia polysaccharide [133]; Green tea [134] ; Grifola frondosa [135]; GRO-beta [136]; GW654652[137]

Heparan sulfate suleparoide [138]; Heparanase [139]; Heparin or heparin fragments+cortisone [57,140-142,285]; HGF-like basic hexapeptides [143]; Herbamycin [144]; Histidine-proline-rich glycoprotein [145]; HIV-1 protease inhibitors [146]; Hox D10 [147]; Homocysteine [148]; HST-1 protein [149]; Human neutrophil peptides [151]; Hydroxycamptothecin [152]; Hyperforin [154]; Hypertermia [155]; Hypoestoxide [156]; Hypoxic eytotoxin TX-402 [157]; Hypoxia cytotoxins [158]

Indinavir and saquinavir [146]; Indolin-2-ketone compound [159]; Inhibitors of basement membrane biosynthesis [160-162]; Inhibitors of DNA methyltransferase [163]; IGF binding protein [164]; IL-12, -18, -21, 27 [166-169]; Ionizing radiation [170]; Isoflavones [171]; Isoprostanes [172]; Isosorbide mono-dinitrate [173]; JNI-17029259 [174]; JNI-26076713 [175]

KIN-841 [176]; Kininogen, kininogen-derived polyptides, kinostatin and related Mab [177-179,200]; KV11 [180]; Lactacystin [181]; Lambda-carragenan oligosaccharide [68]; Laminin-derived peptide [182]; Lamininarin sulphate [183]; Larg-A [184]; Lebectin [185]; Lebestatin [186]; LMW polysaccharide extracts from Agaricus blazei [187]; Lonicera japonica [188]; LMW undersulfated glycol-split heparin [142]; Low sulphated oligosaccharides from heparan sulphate [189]; Lysozime [190]

Marine-derived oligosaccharide sulfate [192]; Metastatin [193]; 2-Methoxyestradiol [194]; Methylene blue [195]; Methyltransferase inhibitors [163]; Microrganism fermantation [196]; Midkine [197]; Mitoxantrone [198]; Mixture of ascorbic acid, lisine, proline and gree tea [199]; Motuporanines [201]; Multiple RTK inhibitors [202]; Mustard essential oil [203]; Myo-inositol trispyrophosphate [204]

Neomycin [205]; Neridronate [206]; Neuregulin-2 [207]; Neurokinin-B [208]; Nitric oxide [173,210]; Nitrotoluene sulfonate [211]; Nonpeptide topomimetics [212]; Notch 4 [213]; Nucleolin antagonist [214]; Obtustatin [215]

Octacosanol [216]; Oncothanin [217]; 5'-O-trityl nucleoside analogs [218]; Opioid peptides [219]; Oriental herbal [220]; Oxaliplatin [221]; Paclitaxel [104]; PAI-1 [222]; PAK1[105]; PE [223]; Pedicularioside G [224]; PGG [225]; Pentosan polysulfate [226]; Pentraxin 3 [227]; Peptide trivalent arsenical [228]; Perillyl alcohol [229]; PPAR agonists [230]; PEX [231]; Phenethyl isothiocyanate [232]; Phenolic compounds [233]; P-henylenabil selenocyanate [234]; Philinopside A [235]; Phorbol esters [236]; Photodynamic therapy [237]; Piperazine derivative [238,239]; Placental ribonuclease inhibitor [240]; Plasma hyaluronan binding protein [241]; Plasminogen related protein [243]; PF4 [244,245]; PARP inhibitor [247]; Poly-L-lisyne/heparin [248]; Polysulphated derivative of tlaminarin [183]; Pomegranate [249]; Prenylnaringenin [250]; Prolactin [150,246]; Proline analogs [251]; Protamine [252]; PAR-1 antagonists [253]; Prothrombin fragments and rh prothrombin kringles [254, 266]; Pyrimidines [255]; Purine analogues [256]; Purine riboside [257]; Pyracoumarin compounds [258]; Pyrazine [259]; p38 MAPK [260]

Quinoline [262]; Radicicol [263]; RDG-peptidomimetic [264]; Rh plasminogen kringle 1-3 [265]; Recombinant kringle domains of plasminogen, tissue-type plasminogen activator and urokinase [267-269]; Red wine [134]; Resveratrol [17]; Retinoids [270]; Rhodostomin [271]; Ribavirin [272]; Ribonuclease inhibitor [273]; Rosiglitazone [274]; Ruthenium red [275]

Safrole oxide [276]; Salmosin [277]; Sangivamycin [278]; Sanguinarine [279]; Saurus chinensis [280]; Sedun sarmentosum [281]; Serpin [282]; Sesterterpenes [283]; PF-4 [284]; Short peptide[286]; Simvastatin [287]; SJ-8002 [288]; S-nitrosocaptopril [289]; Sodium caffeate [290]; Solanum nigrum [291]; Somatostatin [292]; Somocystinamide [293]; Soy isoflavones [294]; S-phosphonate [295]; Spironolactone [296]; Squalamine [297]; Staurosporine [298]; Sulphated GAGs[300]; Sulfated polysaccharide-peptidoglican [301,302]; Sulfonated derivative of dystamycin [303,304]; Sulfonic acid polymers [305]; Sulf-2[306]; Sulindac analogue [307]; Sulindac [308]; Suramin [309-311]; Synthetic Grb2-Src [312]; Synthetic inhibitor of arylsulfatase [211] 
Table 3. Cont.

Taraxacum officinale [314]; Taspine [315]; TAU 1120 [316]; Taxol [317]; Temozolomide [318]; Tenasum-C [319]; Terbinafine [320]; Terpenoids [321]; Tetrac [322]; Tetrameric tripeptide [323]; TGF-b[324]; Thalidomide metabolites [153]; 6-Thioguanine [325]; TSP-1 [326,327]; TP inhibitors [327]; Thymosin peptides [328]; Tinzaparin [329]; TIMP-3 [330]; TFPI [331]; Titanocene dichloride [332]; TNP470+ IFNa [333]; Tocotrienol [334]; Tocotrinol [335]; Topoisomerase inhibitors [336,337]; Topotecan [338]; Torilin [339]; Trapidil [340]; Triamcinolone acetonide [341]; Tricyclodecan-9-yl-xanthate [162]; Triphenylmethanem [50]; Tripterygium wilfordii [342]; Triptolide [343]; Triterpene acids [344]; Trypanosoma cruzi calreticulin [345]; Tyrosine phosphatase inhibitor [346]; TZT-1027 [347]

Ulmus davidiana var.japonica [348]; Undersulfated, LMW glycol-split heparin [349]; Ursodeoxycholic acid [350]; Ursolic and oleanolic acid [344]; Valproic acid [351]; Vanillyl alcohol [352]; VEGI [353]; VASH1B [354]; Vasostatin [245,355]; VEGF-toxin conjugate [356]; Vinblastine+rapamycic [357,358]; Vitamin D binding protein [359]; Vitamin D3 analogues [360]; Vitreous [361]; von Hippel-Lindau protein [362]; Wogonin [363]; Zoledronic acid [364]

\section{* References between brackets.}

\section{Disadvantages of the CAM Assay}

The major disadvantage of CAM is that it already contains a well-developed vascular network and the vasodilation that invariably follows its manipulation may be hard to distinguish from the effects of the test substance. Another limitation in nonspecific inflammatory reaction from the implant. Histologic study of CAM sections demonstrates the presence of perivascular inflammatory infiltrate together with any hyperplastic reaction of the chorionic epithelium. Nonspecific inflammatory reactions are much less frequent when the implant is made very early in CAM development and the host's immune system is relatively immature [6]. Moreover, it might emphasize that species-specific differences might arise if one attempt to test the effects of high affinity antibodies generated against human surface antigens. However, to circumvent this drawback it is useful to perform the experiments early in the CAM development, since at that time the host's immune system is relatively immature [6].

\section{Concluding Remarks}

CAM is widely utilized as an in vivo system to study antiangiogenesis. It offers the advantage of being relatively inexpensive and lends itself to large-scale screening, by using various stimulators alone or in combination with an antiangiogenic agent to examine the effectiveness of an inhibitor. The principal restrictions to its use are essentially due to nonspecific inflammatory reactions and to the presence of pre-existing vessels which make it difficult to determine the true extent of antiangiogenesis.

\section{Acknowledgements}

Supported in part by MIUR (PRIN 2007), Rome, and Fondazione Cassa di Risparmio di Puglia, Bari, Italy.

\section{References}

1. Ribatti, D.; Nico, B.; Crivellato, E.; Roccaro, A.M.; Vacca, A. The history of the angiogenic switch concept. Leukemia 2007, 21, 44-52. 
2. Folkman, J. Tumor angiogenesis: Therapeutic implications. New Engl. J. Med. 1971, 285, $1182-1186$.

3. Nyberg, P.; Xie, L.; Kalluri R. Endogenous inhibitors of angiogenesis. Cancer Res. 2005, 65, 3967-79.

4. Ellis, L.M. Bevacizumab. Nat. Rev. Drug Discov. 2005, S8-S9.

5. Norrby, K. In vivo models of angiogenesis. J. Cell. Mol. Med. 2006, 10, 588-612.

6. Ribatti, D. Chick embryo chorioallantoic membrane as a useful tool to study angiogenesis. Int. Rev. Cell. Mol. Biol. 2008, 270, 181-224.

7. Ribatti, D; Nico, B.; Vacca, A.; Presta, M. The gelatin sponge-chorioallantoic membrane assays. Nat. Prot. 2006, 1, 85-91.

8. Taylor, S.; Folkman, J. Protamine is an inhibitor of angiogenesis. Nature 1982, 297, 307-312.

9. Zacchigna, S.; Zentilin, L.; Morini, M.; Dell'Eva, R.; Noonan, D.M.; Albini, A.; Giacca, M. AAV-mediated gene transfer of tissue inhibitor of metalloproteinases-1 inhibits vascular tumor growth and angiogenesis in vivo. Cancer Gene Ther. 2004, 11, 73-80.

10. Lin, Y.; Wu, X.; Shen, Y.; Bu, P.; Yang, D.; Yan, X. A novel antibody AA98 V(H)/L directed against CD146 efficiently inhibits angiogenesis. Anticancer Res. 2007, 27, 4219-4224.

11. Paris, D.; Townsed, K.; Quadros, A.; Humphrey, J.; Sun, J.; Brem, S.; Wotoczek-Obadia, M.; DelleDonne, A.; Patel, N.; Obregon, D.F.; Crescentini, R.; Abdullah, L.; Coppola, D.; Rojiani, A.M.; Crawford, F.; Sebti, S.M.; Mullan, M. Inhibition of angiogenesis by Abeta peptides. Angiogenesis 2004, 7, 75-85.

12. Rodríguez-Nieto, S.; González-Iriarte, M.; Carmona, R.; Muñoz-Chápuli, R.; Medina, M.A.; Quesada, A.R. Antiangiogenic activity of aeroplysinin-1, a brominated compound isolated from a marine sponge. FASEB J. 2002, 16, 261-263.

13. Brakenhielm, E.; Veitonmaki, N.; Cao, R.; Kihara, S.; Matsuzawa, Y.; Zhivotovsky, B.; Funahashi, T.; Cao, Y. Adiponectin-induced antiangiogenesis and antitumor activity involve caspase-mediated endothelial cell apotosis. Proc. Natl. Acad. Sci. USA 2004, 101, 2476-2481.

14. Li, L.; Yuan, Y.Z.; Lu, J.; Xia, L.; Zhu, Y.; Zhang, Y.P.; Qiao, M.M. Treatment of pancreatic carcinoma by adenoviral mediated gene transfer of vasostatin in mice. Gut. 2006, 55, 259-265.

15. Yeh, C.H.; Wang, W.C.; Hsieh, T.T.; Huang, T.F. Agkistin, a snake venom-derived glycoprotein $\mathrm{Ib}$ antagonist, disrupts von Willebrand factor-endothelial cell interaction and inhibits angiogenesis. J. Biol. Chem. 2000, 275, 18615-18618.

16. Kusaka, M.; Sudo, K.; Fujita, T.; Marui, S.; Itoh, F.; Ingber, D.; Folkman, J. Potent anti-angiogenic action of AGM-1470: Comparison to the fumagillin parent. Biochem. Biophys. Res. Commun. 1991, 174, 1070-1076.

17. Mousa, S.S.; Mousa, S.A. Effect of resveratrol on angiogenesis and platelet/fibrinaccelerated tumor growth in the chick chorioallantoic membrane model. Nutr. Cancer 2005, 52, 59-65.

18. Calzada, M.J.; Zhou, L.; Sipes, J.M.; Zhang, J.; Krutzsch, H.C.; Iruela-Arispe, M.L.; Annis, D.S.; Mosher, D.F.; Roberts, D.D. Alpha4betal integrin mediates selective endothelial cell responses to thrombospondins 1 and 2 in vitro and modulates angiogenesis in vivo. Circ. Res. 2004, 94, $462-470$. 
19. Fu, Y.; Ponce, M.L.; Thill, M.; Yuan P; Wang, N.S.; Csaky, K.G. Angiogenesis inhibition and choroidal neovascularisation suppression by sustained delivery of an integrin antagonist, EMD478761. Invest. Ophtalmol. Vis. Sci. 2007, 48, 5184-5190.

20. Belvisi, L.; Riccioni, T.; Marcellini, M.; Vesci, L.; Chiarucci, I.; Efrati, D.; Potenza, D.; Scolastico, C.; Manzoni, L.; Lombardo, K.; Stasi, M.A.; Orlandi, A., Ciucci, A.; Nico, B.; Ribatti, D.; Giannini, G.; Presta, M.; Carminati, P.; Pisano, C. Biological and molecular properties of a new alpha(v)beta3/alpha(v)beta5 integrin antagonist. Mol. Cancer. Ther. 2005, 4, 1670-1680.

21. Giannopoulou, E.; Katsoris, P.; Kardamakis, D.; Papadimitriou, E. Amifostine inhibits angiogenesis in vivo. J. Pharmacol. Exp. Ther. 2003, 304, 729-737.

22. Knoll, A.; Schmidt, S.; Chapman, M.; Wiley, D.; Bulgrin, J.; Blank, J.; Kirchner, L. A comparison of two controlled-release delivery systems for the delivery of amiloride to control angiogenesis. Microvasc. Res. 1999, 58, 1-9.

23. Bhagwat, S.V.; Ladhdenranta, J.; Giordano, R.; Arap, W.; Pasqualini, R.; Shapiro, L.H. CD13/APN is activated by angiogenic signals and is essential for capillary tube formation. Blood 2001, 97, 652-659.

24. Ingber, D.; Fujita, T.; Kishomoto, S.; Sudo, K.; Kanamaru, T.; Brem, H.; Folkman, J. Angioinhibins: Synthetic analogues of fumagillin which inhibit angiogenesis and suppress tumor growth. Nature 1990, 348, 555-557.

25. Lee, O.H.; Fueyo, J.; Xu, J.; Yung, W.K.; Lemoine, M.G.; Lang, F.F.; Bekele, B.N.; Zhou, X.; Alonso, M.A.; Aldape, K.D.; Fuller, G.N.; Gomez-Manzano, C. Sustained angiopoietin-2 expression disrupts vessel formation and inhibits glioma growth. Neoplasia 2006, 8, 419-428.

26. O'Reilly, M.S.; Holmgren, L.; Shing, Y.; Chen, C.; Rosenthal, R.A.; Moses, M.; Lane, W.S.; Cao, Y.; Sage, E.H.; Folkman, J. Angiostatin: A novel angiogenesis inhibitor that mediates the suppression of metastases by a Lewis lung carcinoma. Cell 1994, 79, 315-328.

27. Brand, M.; Lamandé, N.; Larger, E.; Corvol, P.; Gasc, J.M. Angiotensinogen impairs angiogenesis in the chick chorioallantoic membrane. J. Mol. Med. 2007, 85, 451-460.

28. Célérier, J.; Cruz, A.; Lamandé, N.; Gasc, J.M.; Corvol, P. Angiotensinogen and its cleaved derivatives inhibit angiogenesis. Hypertension 2002, 39, 224-228.

29. Maragoudakis, M.E.; Peristeris, P.; Missirilis, E.; Aletras, A.; Andriopoulou, P.; Haralabopoulos, G. Inhibition of angiogenesis by anthracyclines and titanocene dichloride. Ann. N.Y. Acad. Sci. 1999, 732, 280-293.

30. Drake, C.J.; Cheresh, D.A.; Little, C.D. An antagonist of integrin alpha $\mathrm{v}$ beta 3 prevents maturation of blood vessels during embryonic neovascularization. J. Cell Sci. 1995, 108, 2655-2661.

31. Nishikawa, T.; Akiyama, N.; Kunimasa, K.; Oikawa, T.; Ishizuka, M.; Tsujimoto, M.; Natori, S. Inhibition of in vivo angiogenesis by N-betaalanyl-5-S-glutathionyl-3,4-dihydroxyphenylalanine. Eur. J. Pharmacol. 2006, 539, 151-157.

32. Ishii, T.; Hida, T.; Iinuma, S.; Muroi, M.; Nozaki, Y. TAN-1323 C and D, new concanamycin-group antibiotics; detection of the angiostatic activity with a wide range of macrolide antibiotics. J. Antibiot. 1995, 48, 12-20. 
33. Ribatti, D.; Urbinati, C.; Nico, B.; Rusnati, M.; Roncali, L.; Presta, M. Endogenous basic fibroblast growth factor is implicated in the vascularization of the chick embryo chorioallantoic membrane. Dev. Biol. 1995, 170, 39-49.

34. Vitaliti, A.; Wittmer, M.; Steiner, R.; Wyder, L.; Neri, D.; Klemenz, R. Inhibition of tumor angiogenesis by a single-chain antibody directed against vascular endothelial growth factor. Cancer Res. 2000, 60, 4311-4314.

35. Yan, X.; Lin, Y.; Shen, Y.; Shen, Y.; Yuan, M.; Zhang, Z.; Li, P.; Xia, H.; Li, L.; Luo, D.; Liu, Q.; Mann, K.; Bader, B.L. A novel anti-CD146 monoclonal antibody, AA98, inhibits angiogenesis and tumor growth. Blood 2003 102, 184-191.

36. Pernasetti, F.; Nickel, J.; Clark, D.; Baeuerle, P.A.; Van Epps, D.; Freimark, B. Novel anti-denatured collagen humanized antibody D93 inhibits angiogenesis and tumor growth: An extracellular matrix-based therapeutic approach. Int. J. Oncol. 2006, 29, 1371-1379.

37. Jackson, J.K.; Higo, T.; Hunter, W.L.; Burt, H.M. The antioxidants curcumin and quercetin inhibit inflammatory processes associated with arthritis. Inflamm. Res. 2006, 55, 168-175.

38. Kisker, O.; Onizuka, S.; Banyard, J.; Komiyama, T.; Becker, C.M.; Achilles, E.G.; Barnes, C.M.; O'Reilly, M.S.; Folkman, J.; Pirie-Shepherd, S.R. Generation of multiple angiogenesis inhibitors by human pancreatic cancer. Cancer Res. 2001, 61, 7298-7304.

39. Kim, S.H.; Ahn, S.; Han, J.W.; Lee, H.W.; Lee, H.Y.; Lee, Y.W.; Kim, M.R.; Kim, K.W.; Kim, W.B.; Hong, S. Apicidin is a histone deacetylase inhibitor with anti-invasive and anti-angiogenic potentials. Bichem. Biophys. Res. Commun. 2004, 315, 964-970.

40. Taraboletti, G.; Poli, M.; Dossi, R.; Vanenti, L.; Borsotti, P.; Faircloth, G.T.; Broggini, M.; D'Incalci, M.; Ribatti, D.; Gavazzi, R. Antiangiogenic activity of aplidine, a new agent of marine origin. Br. J. Cancer 2004, 90, 2418-2424.

41. Kim, J.S.; Yu, H.K.; Ahn, J.H.; Lee, H.J.; Hong, S.W.; Jung, K.H.; Chang, S.I.; Hong, Y.K.; Joe, Y.A.; Byun, S.M.; Lee, S.K.; Chung, S.I.; Yoon, Y. Human apolipoprotein(a) kringle V inhibits angiogenesis in vitro and in vivo by interfering with the activation of focal adhesion kinases. Biochem. Biophys. Res. Commun. 2004, 313, 534-540.

42. Kim, H.J.; Koh, P.O.; Kang, S.S.; Paik, W.Y.; Choi, W.S. The localization of dopamine D2 receptor mRNA in the human placenta and the anti-angiogenic effect of apomorphine in the chorionallantoic membrane. Life Science 2001, 68, 1031-1040.

43. Camerino, G.M.; Nicchia, G.P.; Dinardo, M.M.; Ribatti, D.; Svelto, M.; Frigeri, A. In vivo silencing of aquaporin-1 by RNA interference inhibits angiogenesis in the chick embryo chorioallantoic membrane assay. Cell. Mol. Biol. (Noisy-le-grand) 2006, 52, 51-56.

44. Park, I.S.; Kang, S.W.; Shin, Y.J.; Chae, K.Y.; Park, M.O.; Kim, M.Y.; Wheatley, D.N.; Min, B.H. Arginine deiminase: A potential inhibitor of angiogenesis and tumour growth. Br. J. Cancer 2003, 89, 907-914.

45. Mahboobi, S.; Pongratz, H.; Hufsky, H.; Hockemeyer, J.; Frieser, M.; Lyssenko, A.; Paper, D.H.; Bürgermeister, J.; Böhmer, F.D.; Fiebig, H.H.; Burger, A.M.; Baasner, S.; Beckers, T. Synthetic 2-aroylindole derivatives as a new class of potent tubulin-inhibitory, antimitotic agents. J. Med. Chem. 2001, 44, 4535-4553. 
46. Zheng, J.P.; Tang, H.Y.; Chen, X.J.; Yu, B.F.; Xie, J.; Wu, T.C. Construction of recombinant plasmid and prokaryotic expression in E. coli and biological activity analysis of human placenta arresten gene. Hepatobiliary Pancreat. Dis. Int. 2006, 5, 74-79.

47. Huan-huan, C.; Li-Li, Y.; Shang-Bin, L. Artesunate reduces chicken chorioallantoic membrane neovascularisation and exhibits antiangiogenic and apoptotic activity on human microvascular dermal endothelial cell. Cancer Lett. 2004, 211, 163-173.

48. Ashino, H.; Shimamura, M.; Nakajima, H.; Dombou, M.; Kawanaka, S.; Oikawa, T.; Iwaguchi, T.; Kawashima, S. Novel function of ascorbic acid as angiostatic factor. Angiogenesis 2003, 6, 259-269.

49. Shailubhai, K.; Dheer, S.; Picker, D.; Kaur, G.; Sausville, E.A.; Jacob, G.S. Atiprimod is an inhibitor of cancer cell proliferation and angiogenesis. J. Exp. Ther. Oncol. 2004, 4, 267-279.

50. Gagliardi, A.R.; Collins, D.C. Inhibition of angiogenesis by aurintricarboxylic acid. Anticancer Res. 1994, 14, 475-479.

51. Asami, Y.; Kakeya, H.; Komi, Y.; Kojima, S.; Nishikawa, K.; Beebe, K.; Neckers, L.; Osada, H. Azaspirine, a fungal product, inhibits angiogenesis by blocking Raf-1 activation. Cancer Sci. 2008, 99, 1853-1858.

52. van der Schaft, D.W.; Toebes, E.A.; Haseman, J.R.; Mayo, K.H.; Griffioen, A.W. Bactericidal/permeability-increasing protein (BPI) inhibits angiogenesis via induction of apoptosis in vascular endothelial cells. Blood 2000, 96, 176-181.

53. Thakur, A.N.; Thakur, N.L.; Indap, M.M.; Pandit, R.A.; Datar, V.V.; Müller, W.E. Antiangiogenic, antimicrobial, and cytotoxic potential of sponge-associated bacteria. Mar. Biotechnol. N.Y. 2005, 7, 245-252.

54. Liu, J.J.; Huang, T.S.; Cheng, W.F.; Lu, F.J. Baicalein and baicalin are potent inhibitors of angiogenesis: Inhibition of endothelial cell proliferation, migration and differentiation. Int. J. Cancer 2003, 106, 559-565.

55. Oikawa, T.; Hirotani, K.; Ogasawara, H.; Katayama, T., Ashino-Fuse, H.; Shimamura, M.; Iwaguchi, T.; Nakamura, O. Inhibition of angiogenesis by bleomycin and its copper complex. Chem. Pharm. Bull. 1990, 38, 1790-1792.

56. Manolopoulos, V.G.; Liekens, S.; Koolwijk, P.; Voets, T.; Peters, E.; Droogmans, G.; Lelkes, P.I.; De Clercq, E.; Nilius, B. Inhibition of angiogenesis by blockers of volume-regulated anion channels. Gen. Pharmacol. 2000, 34, 107-116.

57. Folkman, J.; Weisz, P.B.; Joullic, M.M.; Li, W.W.; Ewing, W.R. Control of angiogenesis with synthetic heparin substitutes. Science 1989, 243, 1490-1493.

58. Wang, X.H.; Xu, B.; Liu, J.T.; Cui, J.R. Effect of beta-escin sodium on endothelial cells proliferation, migration and apoptosis. Vascul. Pharmacol. 2008, 49, 158-165.

59. Komi, Y.; Suzuki, Y.; Shimamura, M.; Kajimoto, S.; Nakajo, S.; Masuda, M.; Shibuya, M.; Itabe, H.; Shimokado, K.; Oettgen, P.; Nakaya, K.; Kojima, S. Mechanism of inhibition of tumor angiogenesis by beta-hydroxyisovaleryishikonin. Cancer Sci. 2009, 100, 269-277.

60. David, L.; Mallet, C.; Keramidas, M.; Lamandé, N.; Gasc, J.M.; Dupuis-Girod, S.; Plauchu, H.; Feige, J.J.; Bailly, S. Bone morphogenetic protein-9 is a circulating vascular quiescence factor. Circ. Res. 2008, 102, 914-922. 
61. Roccaro, A.M.; Hideshima, T.; Richardson, P.G.; Russo, D.; Ribatti, D.; Vacca, A.; Dammacco, F.; Anderson, K.C. Bortezomib as an antitumor agent. Curr. Pharm. Biotechnol. 2006, 7, 441-448.

62. Gururaj, A.E.; Belakavadi, M.; Salimath, B.P. Antiangiogenic effects of butyric acid involve inhibition of VEGF/KDR gene expression and endothelial cell proliferation. Mol. Cell. Bichem. 2003, 243, 107-112.

63. Kohn, E.C.; Alessandro, R.; Spoonster, J.; Wersto, R.P.; Liotta, L.A. Angiogenesis: Role of calcium-mediated signal transduction. Proc. Natl. Acad. Sci. USA 1995, 92, 1307-1311.

64. Choi, J.M.; Lee, E.O.; Lee, H.J.; Kim, K.H.; Ahn, K.S.; Shim, B.S.; Kim, N.I.; Song, M.C.; Baek, N.I.; Kim, S.H. Identification of campesterol from Chrysanthemum coronarium L. and its antiangigenic activities. Phytother. Res. 2007, 21, 954-959.

65. Hou, W.H.; Wang, T.Y.; Yuan, B.M.; Chai, Y.R.; Jia, Y.L.; Tian, F.; Wang, J.M.; Xue, L.X. Recombinant mouse canstatin inhibits chicken embryo chorioallantoic membrane angiogenesis and endothelial cell proliferation. Acta Biochim. Biophys. Sin. (Shanghai) 2004, 36, 845-850.

66. Min, J.K.; Han, K.Y.; Kim, E.C.; Kim, Y.M.; Lee, S.W.; Kim, O.H.; Kim, K.W.; Gho, Y.S.; Kwon, Y.G. (2004) Capsaicin inhibits in vitro and in vivo angiogenesis. Cancer Res. 2004, 64, 644-651.

67. Murugesan, S.; Mousa, S.A.; O'connor, L.J.; Lincoln, D.W. $2^{\text {nd }}$; Linhardt, R.J. Carbon inhibits vascular endothelial growth factor- and fibroblast growth factor-promoted angiogenesis. FEBS Lett. 2007, 581, 1157-1160.

68. Chen, H.; Yan, X.; Lin, J.; Wang, F.; Xu, W. Depolymerized products of lambda-carrageenan as a potent angiogenesis inhibitor. J. Agric. Food Chem. 2007, 55, 6910-6917.

69. Eisenstein, R.; Kuettner, K.E.; Neopolitan, C.; Soble, L.W.; Sorgente, N. The resistance of certain tissues to invasion. III. Cartilage extracts inhibit the growth of fibroblasts and endothelial cells in culture. Am. J. Pathol. 1975, 87, 337-348.

70. Maiti, T.K.; Chatterjee, J.; Dasgupta, S. Effect of green tea polyphenols on angiogenesis induced by an angiogenin-like protein. Biochem. Biophys. Res. Commun. 2003, 308, 64-67.

71. Vincent, L.; Soria, C.; Mirshahi, F.; Opolon, P.; Mishal, Z.; Vannier, J.P.; Soria, J.; Hong, L. Cerivastatin, an inhibitor of 3-hydroxy-3-methylglutaryl coenzyme a reductase, inhibits endothelial cell proliferation induced by angiogenic factors in vitro and angiogenesis in in vivo models. Arterioscler. Thromb. Vasc. Biol. 2002, 22, 623-629

72. Hussain, S.; Slevin, M.; Mesaik, M.A.; Choudhary, M.I.; Elosta, A.H.; Matou, S.; Ahmed, N.; West, D.; Gaffney, J. Cheiradone: A vascular endothelial cell growth factor receptor antagonist. BMC Cell Biol. 2008, 9, 7.

73. Presta, M.; Oreste, P.; Zoppetti, G.; Belleri, M.; Tanghetti, E.; Leali, D.; Urbinati, C.; Bugatti, A.; Ronca, R.; Nicoli, S.; Moroni, E.; Stabile, H.; Camozzi, M.; Hernandez, G.A.; Mitola, S.; Dell'Era, P.; Rusnati, M.; Ribatti, D. Antiangiogenic activity of semisynthetic biotechnological heparins: Low-molecular-weight-sulfated Escherichia coli K5 polysaccharide derivatives as fibroblast growth factor antagonists. Arterioscler. Thromb. Vasc. Biol. 2005, 25, 71-76.

74. Andrés, G.; Leali, D.; Mitola, S.; Coltrini, D.; Camozzi, M.; Corsini, M.; Belleri, M.; Hirsch, E.; Schwendener, R.A.; Christofori, G.; Alcamí, A.; Presta, M. A Pro-inflammatory Segnature Mediates FGF2-induced angiogenesis. J. Cell. Mol. Med. 2008, (Epub ahead of print). 
75. Hiraki, Y.; Mitsui, K.; Endo, N.; Takahashi, K.; Hayami, T.; Inoue, H.; Shukunami, C.; Tokunaga, K.; Kono, T.; Yamada, M.; Takahashi, H.E.; Kondo, J. Molecular cloning of human chondromodulin-I, a cartilage-derived growth modulating factor, and its expression in Chinese hamster ovary cells. Eur. J. Biochem. 1999, 260, 869-878.

76. Lin, C.M.; Chang, H.; Li, S.Y.; Wu, I.H.; Chiu, J.H. Chrysin inhibits lipopolysaccharide-induced angiogenesis via down-regulation of VEGF/VEGFR-2(KDR) and IL-6/IL-6R pathways. Planta Med. 2006, 72, 708-714.

77. Ejaz, S.; Insan-ud-din; Ashraf, M.; Nawaz, M.; Lim, C.W.; Kim, B. Cigarette smoke condensate and total particulate matter severely distrupts physiological angiogenesis. Food Chem. Toxicol. 2009, 47, 601-614.

78. Ribatti, D.; Maruotti, N.; Nico, B.; Longo, V.; Manieri, D.; Vacca, A.; Cantatore, F.P. Clodronate inhibits angiogenesis in vitro and in vivo. Oncol. Rep. 2008, 19, 1109-1112.

79. Thapa, D.; Lee, J.S.; Park, S.Y.; Bae YH; Bae SK; Kwon, J.B.; Kim, K.J.; Kwak, M.K.; Park, Y.J.; Choi, H.G.; Kim, J.A. Clotrimazole ameliorates intestinal inflammation and abnormal angiogenesis by inhibiting interleukin-8 expression through a nuclear factor-kappaB-dependent manner. J. Pharmacol. Exp. Ther. 2008, 327, 353-364.

80. Zhou, Q.; Nakada, M.T.; Arnold, C.; Shieh, K.Y.; Markland, F.S. Contortrostatin, a dimeric disintegrin from Agkistrodon contortrix contortrix, inhibits angiogenesis. Angiogenesis 1999, 3, 259-269.

81. Gururaj, A.E.; Belakavadi, M.; Venkatesh, D.A.; Marmé, D.; Salimath, B.P. Molecular mechanisms of anti-angiogenic effect of curcumin. Biochem. Biophys. Res. Commun. 2002, 297, 934-942.

82. Hahm, E.R.; Gho, Y.S.; Park, S.; Park, C.; Kim, K.W.; Yang, C.H. Synthetic curcumin analogs inhibit activator protein-1 transcription and tumor-induced angiogenesis. Biochem. Biophys. Res. Commun. 2004, 321, 337-344.

83. Yadav VR; Suresh S,; Devi K; Yadav, S. Novel formulation of solid lipid microparticles of curcumin for anti-angiogenic and anti-infiammatory activity for optimization of therapy of infiammatory bowel disease. J. Pharm. Pharmacol. 2009, 61, 311-321.

84. Brooks, P.C.; Montgomery, A.M.; Rosenfeld, M.; Reisfeld, R.A.; Hu, T.; Klier, G.; Cheresh, D.A. Integrin alpha $\mathrm{v}$ beta 3 antagonists promote tumor regression by inducing apoptosis of angiogenic blood vessels. Cell 1994, 79, 1157-1164.

85. Friedlander, M.; Brooks, P.C.; Shaffer, R.W.; Kincaid, C.M.; Varner, J.A.; Cheresh, D.A. Definition ot two distinct angiogenic pathways by distinct $\alpha \mathrm{v}$ integrins. Science 1995, 270, $1500-1502$.

86. Jung, H.J.; Shimm, J.S.; Suh, Y.G.; Kim, Y.M.; Ono, M.; Kwon, H.J. Potent inhibition of in vivo angiogenesis and tumor growth by a novel cyclooxygenase-2 inhibitor, enoic acanthoic acid. Cancer Sci. 2007, 98, 1943-1948.

87. Park, B.C.; Park, S.Y.; Lee, J.S.; Mousa, S.A.; Kim, J.T.; Kwak, M.K.; Kang, K.W.; Lee, E.S.; Choi, H.G.; Yong, C.S.; Kim, J.A. The anti-angiogenic effects of 1-furan-2-yl-propenone are mediated through the suppression of both VEGF production and VEGF-induced signaling. Vascul. Pharmacol. 2009, 50, 123-131. 
88. Zilberberg, L.; Shinkaruk, S.; Leguin, O.; Rousseau, B.; Hagedorn, M.; Costa, F.; Caronzolo, D.; Balke, M.; Canron, X.; Convert, O.; Laïn, G.; Gionnet, K.; Goncalvès, M., Bayle, M.; Bello, L.; Chassaing, G.; Deleris, G.; Bikfalvi, A. Structure and inhibitory effects on angiogenesis and tumor development of a new vascular endothelial growth inhibitor. J. Biol. Chem. 2003, 278, 35564-35573.

89. Iurlaro, M.; Vacca, A.; Minischetti, M.; Ribatti, D.; Pellegrino, A.; Sardanelli, A.; Giacchetta, F.; Dammacco, F. Antiangiogenesis by cyclosporine. Exp. Hematol. 1998, 26, 1215-1222.

90. Melkonian, G.; Munoz, N.; Chung, J.; Tong, C.; Marr, R.; Talbot, P. Capillary plexus development in the day five to day six chick chorioallantoic membrane is inhibited by cytochalasin D and suramin. J. Exp. Zool. 2002, 292, 241-254.

91. Balzarini, J.; Gamboa, A.E.; Esnouf, R.; Liekens, S.; Neyts, J.; De Clercq, E.; Camarasa, M.J.; Pérez-Pérez, M.J. 7-Deazaxanthine, a novel prototype inhibitor of thymidine phosphorylase. FEBS Lett. 1998, 438, 91-95.

92. Kim, J.H.; Kim JH; Yu, Y.S.; Park, K.H.; Kang, H.J.; Lee, H.Y.; Kim, K.W. Antiangiogenic effect of deguelin on choroidal neovascularization. J. Pharmacol. Exp. Ther. 2008, 324, 643-647.

93. Favot, L.; Martin, S.; Keravis, T.; Andriantsitohaina, R.; Lugnier, C. Involvement of cyclin-dependent pathway in the inhibitory effect of delphinidin on angiogenesis. Cardiovasc. Res. 2003, 59, 479-487.

94. Lee, D.Y.; Kim, S.K.; Kim, S.Y.; Son, D.H.; Nam, J.H.; Kim, I.S.; Park, R.W.; Kim, S.Y.; Byun, Y. Suppression of angiogenesis and tumor growth by orally active deoxycholic acid-heparin conjugate. J. Control. Release 2007, 118, 310-317.

95. Roy, A.M.; Tiwari, K.N.; Parker, W.B.; Secrist, J.A.3 ${ }^{\text {rd }}$; Li, R.; Qu, Z. Antiangiogenic activity of 4'-thio-beta-D-arabinofuranosylcytosine. Mol. Cancer. Ther. 2006, 5, 2218-2224.

96. Takigawa M; Enomoto M; Nishida, Y.; Pan, H.O.; Kinoshita, A.; Suzuki, F. Tumor angiogenesis and polyamines: Alpha-difluoromethylornithine, an irreversible inhibitor of ornithine decarboxylase, inhibits B16 melanoma-induced angiogenesis in ovo and the proliferation of vascular endothelial cells in vitro. Cancer Res. 1990, 50, 4131-4138.

97. Takano, S.; Gately, S.; Jiang, J.B.; Brem, S. A diaminoantraquinone inhibitor of angiogenesis. J. Pharmacol. Exp. Ther. 1994, 271, 1027-1033.

98. Martínez-Poveda, B.; Muñoz-Chápuli, R.; Rodríguez-Nieto, S.; Quintela, J.M.; Fernández, A.; Medina, M.A.; Quesada, A.R. IB05204, a dichloropyridodithienotriazine, inhibits angiogenesis in vitro and in vivo. Mol. Cancer Ther. 2007, 6, 2675-2685.

99. Chen, H.H.; Zhou, H.J.; Wang, W.Q.; Wu, G.D. Antimalarial dihydroartemisinin also inhibits angiogenesis. Cancer Chemother. Pharmacol. 2004, 53, 423-432.

100. Bian, W.; Chen, F.; Bai, L.; Zhang, P.; Qin, W. Dihydrotanshinone I inhibits angiogenesis both in vitro and in vivo. Acta Biochim. Biophys. Sin. (Shanghai) 2008, 40, 1-6.

101. Svensson, A.; Azarbayjani, F.; Bäckman, U.; Matsumoto, T.; Christofferson, R. Digoxin inhibits neuroblastoma tumor growth in mice. Anticancer Res. 2005, 25, 207-212.

102. Martinéz-Poveda, B.; MunPoz-Chápuli, R.; Riguera, R.; Fernández, A.; Medina, M.A.; Quesada, A.R. DTD, an anti-inflammatory ditriazine. Inhibits angiogenesis in vitro and in vivo. J. Cell.Mol. Med. 2008, 12, 1211-1219. 
103. Liu, Y.; Wu, J.; Ho, P.Y.; Chen, L.C.; Chen, C.T.; Liang, Y.C.; Cheng, C.K.; Lee, W.S. Anti-angigenic action of 5,5-diphenyl-2-thiohydantoin-N10 (DPTH-N10). Cancer Lett. 2008, 271, 294-305.

104. Vacca, A.; Ribatti, D.; Iurlaro, M.; Merchionne, F.; Nico, B.; Ria, R.; Dammacco, F. Docetaxel versus paclitaxel for antiangiogenesis. J. Hematother. Stem Cell Res. 2002, 11, 103-118.

105. Kiosses, W.B.; Hood, J.; Yang, S.; Gerritsen, M.E.; Cheresh, D.A.; Alderson, N.; Schwartz, M.A. A dominant negative p65 PAK peptide inhibits angiogenesis. Circ. Res. 2002, 90, 697-702.

106. Garrison, J.B.; Shaw, Y.J.; Chen, C.S.; Kyprianou, N. Novel quinazoline-based compounds impair prostate tumorigenesis by targeting tumor vascularity. Cancer Res. 2007, 67, 11344-11352.

107. Richardson, M.; Wong, D.; Lacroix, S.; Stanisz, J.; Singh, G. Inhibition by doxycycline of angiogenesis in the chicken chorioallantoic membrane (CAM). Cancer Chemother. Pharmacol. 2005, 56, 1-9.

108. Splawinski, J.; Michna, M.; Palczak, R.; Konturek, S.; Splawinska, B. Angiogenesis, quantitative assessment by the chick chorioallantoic membrane assay. Meth. Find. Explt. Clin. Pharmacol. 1988, 10, 221-226.

109. Lirdprapamongkol, K.; Kramb, J.P.; Chokchaichamnankit, D.; Srisomsap, C.; Surarit, R.; Sila-Asna, M.; Bunyaratvej, A.; Dannhardt, G.; Svasti, J. Juice of eclipta prostrata inhibits cell migration in vitro and exhibits anti-angiogenic activity in vivo. In Vivo 2008, 22, 363-368.

110. Kwak, H.J.; Park, M.J.; Park, C.M.; Moon, S.I.; Yoo, D.H.; Lee, H.C.; Lee, S.H.; Kim, M.S.; Lee, H.W.; Shin, W.S.; Park, I.C.; Rhee, C.H.; Hong, S.I. Emodin inhibits vascular endothelial growth factor-A-induced angiogenesis by blocking receptor-2 (KDR/Flk-1) phosphorylation. Int. J. Cancer 2006, 118, 2711-2720.

111.Pisanti, S.; Borselli, C.; Oliviero, O.; Laezza, C.; Gazzerro, P.; Bifulco, M. Antiangiogenic activity of the endocannabinoid anandamide: Correlation to its tumor-suppressor efficacy. J. Cell Physiol. 2007, 211, 495-503.

112. Mongiat, M.; Sweeney, S.M.; San Antonio, J.D.; Fu, J.; Iozzo, R.V. Endorepellin, a novel inhibitor of angiogenesis derived from the $\mathrm{C}$ terminus of perlecan. J. Biol. Chem. 2002, 278, 4238-4249.

113. Ling, Y.; Yang, Y.; Lu, N.; You, Q.D.; Wang, S.; Gao, Y.; Chen, Y.; Guo, Q.L. Endostar, a novel recombinant human endostatin, exerts antiangiogenic effect via blocking VEGF-induced tyrosine phosphorylation of KDR/FLK-1 of endothelial cells. Biochem. Biophys. Res. Commun. 2007, 361, 79-84.

114. Liu, F.; Tan, G.; Li, J.; Dong, X.; Krissansen, G.W.; Sun, X. Gene transfer of endostatin enhances the efficacy of doxorubicin to suppress human hepatocellular carcinomas in mice. Cancer Sci. 2007, 98, 1381-1387.

115. Oikawa, T.; Haegawa, M.; Shimamura, M.; Ashino, H.; Murota, S.; Morita, I. Eponeomycin, a novel antibiotic, is a high powerful angiogenesis inhibitor. Biochem. Biophys. Res. Commun. 1993, 181, 1070-1076.

116. Michaelis, U.R.; Fisslthaler, B.; Barbosa-Sicard, E.; Falck, J.R.; Fleming, I.; Busse, R. Cytochrome P450 epoxygenases 2C8 and 2C9 are implicated in hypoxia-induced endothelial cell migration and angiogenesis. J. Cell Sci. 2005, 118, 5489-5498.

117. Gagliardi, A.R.; Collins, D.C. Inhibition of angiogenesis by antiestrogens. Cancer Res. 1993, 53, 533-535. 
118. Forough, R.; Weylie, B.; Collins, C.; Parker JL; Zhu J; Barhoumi R; Watson, D.K. Transcription factor Ets-1 regulates fibroblast growth factor-1-mediated angiogenesis in vivo: Role of Ets-1 in the regulation of the PI3K/AKT/MMP-1 pathway. J. Vasc. Res. 2006, 43, 327-337.

119. Wernert, N.; Stanjek, A.; Hügel, A.; Giannis, A. Inhibition of angiogenesis on the chicken chorioallantoic membrane by Ets 1 antisense oligodeoxyribonucleotides. Verh. Dtsch. Ges. Pathol. 1999, 83, 212-215.

120. Shyu, K.G.; Lin, S.; Lee, C.C.; Chen, E.; Lin, L.C.; Wang, B.W.; Tsai, S.C. Evodiamine inhibits in vitro angiogenesis: Implication for antitumorgenicity. Life Sci. 2006, 78, 2234-2243.

121. Lin, J.; Yan, X.J.; Chen, H.M. Fascaplysin, a selective CDK4 inhibitor, exhibit anti-angiogenic activity in vitro and in vivo. Cancer Chemoter. Pharmacol. 2007, 59, 439-445.

122. Ribatti, D.; Nico, B.; Morbidelli, L.; Donnini, S.; Ziche, M.; Vacca, A.; Roncali, L.; Presta, M. Cell-mediated delivery of fibroblast growth factor-2 and vascular endothelial growth factor onto the chick chorioallantoic membrane: Endothelial fenestrations and angiogenesis. J. Vasc. Res. 2001, 38, 389-397.

123. Lindsay, C.K.; Gomez, D.E.; Thorgeirsson, U.P. Effect of flavone acetic acid on endothelial cell proliferation: Evidence for antiangiogenic properties. Anticancer Res. 1996, 16, 425-431.

124. Igarashi, Y.; Yabuta, Y.; Sekine, A.; Fujii K; Harada, K.; Oikawa, T.; Sato, M.; Furumai, T.; Oki, T. Directed biosynthesis of fluorinated pseurotin A, synerazol and gliotoxin. J. Antibiot. (Tokyo) 2004, 57, 748-754.

125. Ryu, J.; Lee, C.W.; Hong, K.H.; Shin, J.A.; Lim, S.H.; Park, C.S.; Shim, J.; Nam, K.B.; Choi, K.J.; Kim, Y.H.; Han, K.H. Activation of fractalkine/CX3CR1 by vascular endothelial cells induces angiogenesis through VEGF-A/KDR and reverses hindlimb ischaemia. Cardiovasc. Res. 2008, 78, 333-340.

126. Chung, T.W.; Kim, S.J.; Choi, H.J.; Kim, K.J.; Kim, M.J.; Kim, S.H.; Lee, H.J.; Ko, J.H.; Lee, Y.C.; Suzuki, A.; Kim, C.H. Ganglioside GM3 inhibits VEGF/VEGFR-2-mediated angiogenesis: Direct interaction of GM3 with VEGFR-2. Glycobiology 2009, 19, 229-239.

127. Ahn, E.K.; Jeon, H.J.; Lim, E.J.; Jung, H.J.; Park, E.H. Anti-inflammatory and anti-angiogenic activities of Gastrodia elata Blume. J. Ethnopharmacol. 2007, 110, 476-482.

128. Koo, H.J.; Song, Y.S.; Kim, H.J.; Lee, Y.H.; Hong, S.M.; Kim, S.J.; Kim, B.C.; Jin, C.; Lim, C.J.; Park, E.H. Antiinflammatory effects of genipin, an active principle of gardenia. Eur. J. Pharmacol. 2004, 495, 201-208.

129. Conconi, M.T.; Nico, B.; Guidolin, D.; Baiguera, S.; Spinazzi, R.; Rebuffat, P.; Malendowicz, L.K.; Vacca, A.; Carraro, G.; Parnigotto, P.P.; Nussdorfer, G.G.; Ribatti, D. Ghrelin inhibits FGF-2-mediated angiogenesis in vitro and in vivo. Peptides 2004, 25, 2179-2185.

130. Chow, L.M.; Chui, C.H.; Tang, J.C.; Lau, F.Y.; Yau, M.Y.; Cheng, G.Y.; Wong, R.S.; Lai, P.B.; Leung, T.W.; Teo, I.T.; Cheung, F.; Guo, D.; Chan, A.S. Anti-angiogenic potential of Gleditsia sinensis fruit extract. Int. J. Mol. Med. 2003, 12, 269-273.

131. Amin, K.; Li, J.; Chao, W.R.; Dewhirst, M.W.; Haroon, Z.A. Dietary glycine inhibits angiogenesis during wound healing and tumor growth. Cancer Biol. Ther. 2003, 2, 173-178.

132. Abe, M.; Jnoue, D.; Matsunaga, K.; Ohizumi, Y.; Ueda, H.; Asano, T.; Murakami, M.; Sato,Y. Goniodomin A, an antifungal polyether macrolide, exhibits antiangiogenic activities via inhibition of actin reorganization in endothelial cells. J. Cell Physiol. 2002, 190, 109-116. 
133. Zhang, C.; Yang, F.; Zhang, X.W.; Wang, S.C.; Li, M.H.; Lin, L.P.; Ding, J. Grateloupia longifolia polysaccharide inhibits angiogenesis by downregulating tissue factor expression in HMEC-1 endothelial cells. Br. J. Pharmacol. 2006, 148, 741-751.

134. Oak, M.H.; El Bedoui, J.; Schini-Kerth, V.B. Antiangiogenic properties of natural polyphenols from red wine and green tea. J. Nutr. Biochem. 2005, 16, 1-8.

135. Lee, J.S.; Park, B.C.; Ko, Y.J.; Choi, M.K.; Choi, H.G.; Yong, C.S.; Lee, J.S.; Kim, J.A. Grifola frondosa (maitake mushroom) water extract inhibits vascular endothelial growth factor-induced angiogenesis through inhibition of reactive oxygen species and extracellular signal-regulated kinase phosphorylation. J. Med. Food 2008, 11, 643-651.

136. Cao, Y.; Chen, C.; Weatherbee, J.A.; Tsang, M.; Folkman, J. GRO-beta, a -C-X-C- chemokine, is an angiogenesis inhibitor that suppresses the growth of Lewis lung carcinoma in mice. J. Exp. Med. 1995, 182, 2069-2077.

137. Huh, J.I.; Calvo, A.; Stafford, J.; Cheung, M.; Kumar, R.; Philp, D.; Kleinman, H.K., Green, J.E. Inhibition of VEGF receptors significantly impairs mammary cancer growth in C3(1)/Tag transgenic mice through antiangiogenic and non-antiangiogenic mechanisms. Oncogene 2005, 24, 790-800.

138. Benelli, U.; Bocci, G.; Danesi, R.; Lepri, A.; Bernardini, N.; Bianchi, F.; Lupetti, M.; Dolfi, A.; Campagni, A.; Agen, C.; Nardi, M.; Del Tacca, M. The heparan sulfate suleparoide inhibits rat corneal angiogenesis and in vitro neovascularization. Exp. Eye. Res. 1998, 67, 133-142.

139. Sasisekharon, R.; Moses, M.A.; Nugent, M.A.; Cooney, C.L.; Langer, R. Heparinase inhibits neovascularization. Proc. Natl. Acad. Sci. USA 1994, 91, 1524-1528.

140. Folkman J; Langer R; Linhrdt RC; Haudenschild, C.; Taylor, S. Angiogenesis inhibition and tumor regression caused by heparin or a heparin fragment in the presence of cortisone. Science 1983, 221, 719-725.

141. Crum, R.; Szabo, S.; Folkman, J. A new class of steroid inhibit angiogenesis in the presence of heparin or a heparin fragment. Science 1985, 230, 1375-1378.

142. Casu, B.; Guerrini, M.; Guglieri, S.; Naggi, A.; Perez, M.; Torri, G.; Cassinelli, G.; Ribatti, D.; Carminati, P.; Giannini, G.; Penco, S.; Pisano, C.; Belleri, M.; Rusnati, M.; Presta, M. Undersulfated and glycol-split heparins endowed with antiangiogenic activity. J. Med. Chem. 2004, 47, 838-848.

143. Fazekas, K.; Janovics, A.; Döme, B.; Koska, P.; Albini, A.; Tímár, J. Effect of HGF-like basic hexapeptides on angiogenesis. Microvasc. Res. 2001, 62, 440-444.

144. Oikawa, T.; Hirotani, K.; Shimamura, M.; Ashino-Fuse, H.; Iwaguchi, T. Powerful antiangiogenic activity of herbamycin (named angiostatic antibiotic). J. Antibiot. 1989, 42, 1202-1204.

145. Juarez, J.C.; Guan, X.; Shipulina, N.V.; Plunkett, M.L.; Parry, G.C.; Shaw, D.E.; Zhang, J.C.; Rabbani, S.A.; McCrae, K.R.; Mazar, A.P.; Morgan, W.T.; Doñate, F. Histidine-proline-rich glycoprotein has potent antiangiogenic activity mediated through the histidine-proline-rich domain. Cancer Res. 2002, 62, 5344-5350.

146. Sgadari, C.; Barillari, G.; Toschi, E.; Carlei, D.; Bacigalupo, I.; Baccarini, S.; Palladino, C.; Leone, P.; Bugarini, R.; Malavasi, L.; Cafaro, A.; Falchi, M.; Valdembri, D.; Rezza, G.; Bussolino, F.; Monini, P.; Ensoli, B. HIV protease inhibitors are potent anti-angiogenic molecole and promote regression of Kaposi sarcoma. Nat. Med. 2002, 8, 225-232. 
147. Myers, C.; Charboneau, A.; Cheung I ; Hanks, D.; Boudreau, N. Sustained expression of homeobox D10 inhibits angiogenesis. Am. J. Pathol. 2002, 161, 2099-2109.

148. Nagai, Y.; Tasaki, H.; Takatsu, H.; Nihei, S.; Yamashita, K.; Toyokawa, T.; Nakashima, Y. Homocystein inhibits angiogenesis in vitro and in vivo. Biochem. Biophys. Res. Commun. 2001, 281, 726-731.

149. Yoshida, T.; Ishimaru, K.; Sakamoto, H.; Yokota, J.; Hirohashi, S.; Igarashi, K.; Sudo, K.; Terada, M. Angiogenic activity of the recombinant hst-1 protein. Cancer Lett. 1994, 83, 261-268.

150. Ueda, E.; Ozerdem, U.; Chen, Y.H.; Yao, M.; Huang, K.T.; Sun, H.; Martins-Green, M.; Bartolini, P.; Walker AM. A molecular mimic demonstrates that phosphorylated human prolactin is a potent anti-angiogenic hormone. Endocr. Relat. Cancer 2006, 13, 95-111.

151. Chavakis, T.; Cines, D.B.; Rhee, J.S.; Liang, O.D.; Schubert, U.; Hammes, H.P.; Higazi, A.A.; Nawroth, P.P.; Preissner, K.T.; Bdeir, K. Regulation of neovascularization by human neutrophil peptides (alpha-defensins): A link between inflammation and angiogenesis. FASEB J. 2004, 18, 1306-1308.

152. Xiao, D.; Tan, W.; Li, M.; Ding, J. Antiangiogenic potential of 10-hydroxycamptothecin. Life Sci. 2001, 69, 1619-1628.

153. Marks, M.G.; Shi, J.; Fry, M.O.; Xiao, Z.; Trzyna, M.; Pokala, V.; Ihnat, M.A.; Li, P.K. Effects of putative hydroxylated thalidomide metabolites on blood vessel density in the chorioallantoic membrane (CAM) assay and on tumor and endothelial cell proliferation. Bio. Pharm. Bull. 2002, 25, 597-604.

154. Martínez-Poveda, B.; Queseda, A.R.; Medina, M.A. Hyperforin, a bio-active compound of St. John's Wort, is a new inhibitor of angiogenesis targeting several key steps of the process. Int. J. Cancer 2005, 117, 775-780.

155. Roca, C.; Primo, L.; Valdembri, D.; Cividalli, A.; Declerck, P.; Carmeliet, P.; Gabriele, P.; Bussolino, F. Hyperthermia inhibits angiogenesis by a plasminogen activator inhibitor 1-dependent mechanism. Cancer Res. 2003, 63, 1500-1507.

156. Ojo-Amaize, E.A.; Nchekwube, E.J.; Cottam, H.B.; Bai, R.; Verdier-Pinard, P.; Kakkanaiah, V.N.; Varner, J.A.; Leoni, L.; Okogun, J.I.; Adesomoju, A.A.; Oyemade, O.A.; Hamel, E. Hypoestoxide, a natural nonmutagenic diterpenoid with antiangiogenic and antitumor activity: Possible mechanisms of action. Cancer Res. 2002, 62, 4007-4014.

157. Nagasawa, H.; Mikamo, N.; Nakajima, Y.; Matsumoto, H.; Uto, Y.; Hori, H. Antiangiogenic hypoxic cytotoxin TX-402 inhibits hypoxia-inducible factor 1 signaling pathway. Anticancer Res. 2003, 23, 4427-4434.

158. Nagasawa, H.; Yamashita, S.; Mikamo, N.; Shimamura, M.; Oka, S.; Uto, Y.; Hori, H. Design, synthesis and biological activities of antiangiogenic hypoxic cytotoxin, triazine-N-oxide derivatives. Comp. Biochem. Physiol. A. Mol .Integr. Physiol. 2002, 132, 33-40.

159. Wang, L.L.; Li, J.J.; Zheng, Z.B.; Liu, H.Y.; Du, G.J.; Li, S. Antitumor activities of a novel indolin-2-ketone compound, Z24: More potent inhibition on bFGF-induced angiogenesis and bcl-2 over-expressing cancer cells. Eur. J. Pharmacol. 2004, 502, 1-10.

160. Maragoudakis, M.E.; Sarmonika, M.; Panoutscacopoulou, M. Inhibition of basement membrane biosynthesis prevents angiogenesis. J. Pharmacol. Exp. Ther. 1988, 244, 729-733. 
161. Maragoudakis, M.E.; Sarmonika, M.; Panoutscacopoulou, M. Antiangiogenic action of heparin plus cortisone is associated with decreased collagenous protein synthesis in the CAM system. Pharmacol. Exp. Ther. 1989, 251, 679-682.

162. Maragoudakis, M.E.; Missirlis, E.; Sarmonika, M.; Panoutsacopoulou, M.; Karakiulakis, G. Basement membrane biosynthesis as a target tot umor therapy. J. Pharmacol. Exp. Ther. 1990, 252, 753-757.

163. Hellebrekers, D.M.; Jair, K.W.; Viré, E.; Eguchi, S.; Hoebers, N.T.; Fraga, M.F.; Esteller, M.; Fuks, F.; Baylin, S.B.; van Engeland, M.; Griffioen, A.W. Angiostatic activity of DNA methyltransferase inhibitors. Mol. Cancer Ther. 2006, 5, 467-475.

164. Oh, S.H.; Kim, W.Y.; Kim, J.H.; Younes, M.N.; El-Naggar, A.K.; Myers, J.N.; Kies, M.; Cohen, P.; Khuri, F.; Hong, W.K.; Lee, H.Y. Identification of insulin-like growth factor binding protein-3 as a farnesyl transferase inhibitor SCH66336-induced negative regulator of angiogenesis in head and neck squamous cell carcinoma. Clin. Cancer Res. 2006, 12, 653-661.

165. Kumar, C.C.; Malkowski, M.; Yin, Z.; Tanghetti, E.; Yaremko, B.; Nechuta, T.; Varner, J.; Liu, M.; Smith, E.M.; Neustadt, B.; Presta, M.; Armstrong, L. Inhibition of angiogenesis and tumor growth by SCH221153, a dual alpha(v)beta3 and alpha(v)beta5 integrin receptor antagonist. Cancer Res. 2001, 61, 2232-2238.

166. Airoldi, I.; Di Carlo, E.; Cocco, C.; Taverniti, G.; D'Antuono, T.; Ognio, E.; Watanabe, M.; Ribatti, D.; Pistoia, V. Endogenous IL-12 triggers an antiangiogenic program in melanoma cells. Proc. Natl. Acad. Sci. USA 2007, 104, 3996-4001.

167. Cao, R.; Farnebo, J.; Kurimoto, M.; Cao, Y. Interleukin-18 acts as an angiogenesis and tumor suppressor. FASEB J. 1999, 13, 2195-2202.

168. Castermans, K.; Tabruyn, S.P.; Zeng, R.; van Beijnum, J.R.; Eppolito, C.; Leonard, W.J.; Shrikant, P.A.; Griffioen, A.W. Angiostatic activity of the antitumor cytokine interleukin 21. Blood 2008, 112, 4940-4947.

169. Shimizu, M.; Shimamura, M.; Owaki, T.; Asakawa, M.; Fujita, K.; Kudo, M.; Iwakura, Y.; Takeda, Y.; Luster, A.D.; Mizuguchi, J.; Yoshimoto, T. Antiangiogenic and antitumor activity of IL-27. J. Immunol. 2006, 176, 7317-7324.

170. Karnabatidis, D.; Dimopoulos, J.; Siabilis, D.; Papazafiropoulos, D.; Kalogeropoulou, C.P.; Nikiforidis, G. Quantification of the ionising radiation effect over angiogenesis in the chick embryo chorionallantoic membrane by computerised analysis of angiographic images. Acta Radiologica 2001, 42, 333-338.

171. Kiriakidis, S.; Högemeir, O.; Starcke, S.; Dombrowski, F.; Hahne, J.C.; Pepper, M.; Jha, H.C.; Wernert, $\mathrm{N}$. Novel tempeh (fermented soyabean) isoflavones inhibit in vivo angiogenesis in the chicken chorioallantoic membrane assay. Br. J. Nutr. 2005, 93, 317-323.

172. Benndorf, R.A.; Schwedhelm, E.; Gnann, A.; Taheri, R.; Kom, G.; Didié, M.; Steenpass, A.; Ergün, S.; Böger, R.H. Isoprostanes inhibit vascular endothelial growth factor-induced endothelial cell migration, tube formation, and cardiac vessel sprouting in vitro, as well as, angiogenesis in vivo via activation of the thromboxane $\mathrm{A}(2)$ receptor: A potential link between oxidative stress and impaired angogenesis. Circ. Res. 2008, 103, 1037-1046. 
173. Pipili-Synetos, E.; Sakkoula, E.; Haralabopoulos, G.; Andriopoulou, P., Peristeris, P.; Maragoudakis, M.E. Evidence that nitric oxide is an endogenous antiangiogenic mediator. $R$. $J$. Pharmacol. 1995, 111, 894-902.

174. Emanuel, S.; Gruninger, R.H.; Fuenters-Pesquera, A.; Connolly, P.J.; Seamon, J.A.; Hazel, S.; Tominovich, R.; Hollister, B.; Napier, C.; D'Andrea, M.R.; Reuman, M.; Bignan, G.; Tuman, R.; Johnson, D.; Moffatt, D.; Batchelor, M.; Foley, A.; O'Connell, J.; Allen, R.; Perry, M.; Jolliffe, L.; Middleton, S.A. A vascular endothelial growth factor receptor-2 kinase inhibitor potentiates the activity of the conventional chemotherapeutic agents paclitaxel and doxorubicin in tumor xenograft models. Mol. Pharmacol. 2004, 66, 635-647.

175. Santulli, R.J.; Kinney, W.A.; Ghosh, S.; Decorte, B.L.; Liu, L.; Tuman, R.W.; Zhou, Z.; Huebert, N.; Bursell, S.E.; Clermont, A.C.; Grant, M.B.; Shaw, L.C.; Mousa, S.A.; Galemmo, R.A. Jr.; Johnson, D.L.; Maryanoff, B.E.; Damiano, B.P. Studies with an orally bioavailable alpha V integrin antagonist in animal models of ocular vasculopathy: Retinal neovascularization in mice and retinal vascular permeability in diabetic rats. J. Pharmacol. Exp. Ther. 2008, 324, 894-901.

176. Shimamura, M.; Nagasawa, H.; Ashino, H.; Yamamoto, Y.; Hazato, T.; Uto, Y.; Hori, H.; Inayama, S. A novel hypoxia-dependent 2-nitroimidazole $\mathrm{KIN}-841$ inhibits tumour-specific angiogenesis by blocking production of angiogenic factors. Br. J. Cancer 2003, 88, 307-313.

177. Colman, R.W. Regulation of angiogenesis by the kallikrein-kinin system. Curr. Pharm. Des. 2006, 12, 2599-2607.

178. Zhang, J.C.; Qi, X.; Juraz, J.; Plunkett, M.; Donaté, F.; Sakthivel, R.; Mazar, A.P.; McCrae, K.R. Inhibition of angiogenesis by two-chain high molecular weight kininogen (HKa) and kininogen-derived polyptides. Can. J. Physiol. Pharmacol. 2002, 80, 85-90.

179. Colman, R.W.; Iameson, B.A.; Lin, Y.; Johnson, D.; Mousa, S.A. Domain 5 of high molecular weight kininogen (kininostatin) down-regulates endothelial cell proliferation and migration and inhibits angiogenesis. Blood 2000, 95, 543-550.

180. Yi, Z.F.; Cho, S.G.; Zhao, H.; Wu, Y.Y.; Luo, J.; Li, D.; Yi, T.; Xu, X.; Wu, Z.; Liu, M. A novel peptide from human apolipoprotein(a) inhibits angiogenesis and tumor growth by targeting c-Src phosphorylation in VEGF-induced human umbilical endothelial cells. Int. J. Cancer 2009, 124, 843-852.

181. Oikawa, T.; Sasaki, T.; Nakamura, M.; Shimamura, M.; Tanahashi, N.; Omura, S.; Tanaka, K. The proteasoma is involved in angiogenesis. Biochem. Biophys. Res. Commun. 1998, 246, 243-248.

182. Sakamoto, N.; Iwahana, M.; Tanaka, N.G.; Osada, Y. (1991) Inhibition of angiogenesis and tumor growth by a synthetic laminin peptide, CDPGYIGSR-NH2. Cancer Res. 1991, 51, 903-906.

183. Hoffman, R.; Paper, D.H.; Donaldson, J.; Vogl, H. Inhibition of angiogenesis and murine tumour growth by laminarin sulphate. Brit. J. Cancer 1996, 73, 1183-1186.

184. Dias, P.F.; Siqueira, J.M., Jr.; Vendruscolo, L.F.; de Jesus Neiva, T.; Gagliardi, A.R.; Maraschin, M.; Ribeiro-do-Valle, R.M. Antiangiogenic and antitumoral properties of a polysaccharide isolated from the seaweed Sargassum stenophyllum. Cancer Chemother. Pharmacol. 2005, 56, 436-446. 
185. Pilorget, A.; Conesa, M.; Sarray, S.; Michaud-Levesque, J.; Daoud, S.; Kim, K.S.; Demeule, M.; Marvaldi, J.; El Ayeb, M.; Marrakchi, N.; Béliveau, R.; Luis, J. Lebectin, a Macrovipera lebetina venom-derived C-type lectin, inhibits angiogenesis both in vitro and in vivo. J. Cell. Physiol. 2007, $211,307-315$.

186. Olfa, K.Z.; José, L.; Salma, D.; Amine, B.; Najet, S.A.; Nicolas, A.; Maxime, L.; Raoudha, Z.; Kamel, M.; Jacques, M.; Jean-Marc, S.; Mohamed el, A.; Naziha, M. Lebestatin, a disintegrin from Macrovipera venom, inhibits integrin-mediated cell adhesion, migration and angiogenesis. Lab. Invest. 2005, 85, 1507-1516.

187. Niu, Y.C.; Liu, J.C.; Zhao, X.M.; Wu, X.X. A low molecular weight polysaccharide isolated from Agaricus blazei suppresses tumor growth and angiogenesis in vivo. Oncol. Rep. 2009, 21, $145-152$.

188. Yoo, H.J.; Kang, H.J.; Song, Y.S.; Park, E.H.; Lim, C.J. Anti-angiogenic, antinociceptive and anti-inflammatory activities of Lonicera japonica extract. J. Pharm. Pharmacol. 2008, 60, 779-786.

189. Hahnenberger, R.; Jakobson, A.M.; Ansari, A.; Wehler, T.; Svahn, C.M.; Lindahl, U. Low-sulphated oligosaccharides derived from heparan sulphate inhibit normal angiogenesis. Glycobiology 1993, 3, 567-573.

190. Ye, J.; Wang, C.; Chen, X.; Guo, S.; Sun, M. Marine lysozyme from a marine bacterium that inhibits angiogenesis and tumor growth. Appl. Microbiol. Biotechnol. 2008, 77, 1261-1267.

191. Melkonian, G.; Cheung, L.; Marr, R.; Tong, C.; Talbot, P. Mainstream and sidestream cigarette smoke inhibit growth and angiogenesis in the day 5 chick chorioallantoic membrane. Toxicol. Sci. 2002, 68, 237-248.

192. Ma, J.; Xin, X.; Meng, L.; Tong, L.; Lin, L.; Geng, M.; Ding, J. The marine-derived oligosaccaride sul fate (MdOS), anovel multiple tyrosine kinase inhibitor, combats tumor angiogenesis both in vitro and in vivo. PloS ONE 2008, 3, e3774.

193. Liu, N.; Lapcevich, R.K.; Underhill, C.B.; Han, Z.; Gao, F.; Swartz, G.; Plum, S.M.; Zhang, L.; Green, S.J. Metastatin: A hyaluronan-binding complex from cartilage that inhibits tumor growth. Cancer Res. 2001, 61, 1022-1028.

194. D’Amato, R.J.; Lin, C.M.; Flynn, E.; Folkman, J.; Hamel, E. 2-Methoxyestradiol, an endogenous mammalian metabolita, inhibits tubulin polymerization by interacting at the colchicine site. Proc. Natl. Acad. Sci. USA 1994, 91, 3964-3968.

195. Zacharakis, N.; Tone, P.; Flordellis, C.S.; Maragoudakis, M.E.; Tsopanoglou, N.E. Methylene blue inhibits angiogenesis in chick chorioallantoic membrane through a nitric oxide-independent mechanism. J. Cell. Mol. Med. 2006, 10, 493-548.

196. Chui, C.H.; Gambari, R.; Lau, F.Y.; Hau, DK.; Wong, R.S.; Cheng, G.Y.; Kok, S.H.; Higa, T.; Ke, B.; Chan, A.S.; Fong, D.W.; Tang, J.C. Antiangiogenic activity of a concentrated effective microorganism fermentation extract. Int. J. Mol. Med. 2006, 18, 975-979.

197. Van der Horst, E.H.; Leupold, J.H.; Schubbert, R.; Ullrich, A.; Allgayer, H. TaqMan-based quantification of invasive cells in the chick embryo metastasis assay. Biotechniques 2004, 37, 940-945. 
198. Iigo, M.; Shimamura, M.; Sagawa, K.; Tsuda, H. Characteristics of the inhibitory effect of mitoxantrone and pirarubicin on lung metastases of colon carcinoma 26. Jpn. J. Cancer Res. 1995, 86, 867-872.

199. Roomi, M.W.; Roomi, N.; Ivanov, V.; Kalinovsky, T.; Niedzwiecki, A.; Rath, M. Inhibitory effect of a mixuture containing ascorbic acid, lysine, proline and green tea extract on critical parameters in angiogenesis. Oncol. Rep. 2005, 14, 807-815.

200. Colman, R.W. Inhibition of angiogenesis by a monoclonal antibody to kininogen as well as by kininostatin which block proangiogenic high molecular weight kininogen. Int. Immunopharmacol. 2002, 2, 1887-1894.

201. Roskelly, C.D.; Williams, D.E.; McHardy, L.M.; Leong KG; Troussard, A.; Karsan, A.; Andersen, R.J.; Dedhar, S.; Roberge, M. Inhibition of tumor cell invasion and angiogenesis by motuporamines. Cancer Res. 2001, 61, 6788-6794.

202. Gangjee, A.; Namjoshi, O.A.; Yu, J.; Ihnat, M.A.; Thorpe, J.E.; Warnke, L.A. (2008) Design, synthesis and biological evaluation of substituted pyrrol[2,3-d]pyrimidines as multiple receptor tyrosine kinase inhibitors and antiangiogenic agents. Bioorg. Med. Chem. 2008, 16, 5514-5528.

203. Kumar, A.; D’Souza, S.S.; Tickoo, S.; Salimath, B.P.; Singh, H.B. Antiangiogenic and proapoptotic activities of allyl isothiocyanate inhibit ascites tumor growth in vivo. Integr. Cancer Ther. 2009, 8, 75-87.

204. Sihn, G.; Walter, T.; Klein, J.C.; Queguiner, I.; Iwao, H.; Nicolau, C.; Lehn, J.M.; Corvol, P.; Gasc, J.M. Anti-angiogenic properties of myo-inositol trispyrophosphate in ovo and growth reduction of implanted glioma. FEBS Lett. 2007, 581, 962-966.

205. Hu, G.F. Neomycin inhibits angiogenin-induced angiogenesis. Proc. Natl. Acad. Sci. USA 1998, 95, 9791-9795.

206. Ribatti, D.; Nico, B.; Mangieri, D.; Maruotti, N.; Longo, V.; Vacca, A.; Cantatore, F.P. Neridronate inhibits angiogenesis in vitro and in vivo. Clin. Rheumatol. 2007, 26, 1094-1098.

207. Nakano, N.; Higashiyama, S.; Ohmoto, H.; Ishiguro, H.; Taniguchi, N.; Wada, Y. The N-terminal region of NTAK/neuregulin-2 isoforms has an inhibitory activity on angiogenesis. J. Biol. Chem. 2004, 279, 11465-11470.

208. Pal, S.; Wu, J.; Murray, J.K.; Gellman, S.H.; Wozniak, M.A.; Keely, P.J.; Boyer, M.E.; Gomez, T.M.; Hasso, S.M.; Fallon, J.F.; Bresnick, E.H. An antiangiogenic neurokinin-B/thromboxane A2 regulatory axis. J. Cell Biol. 2006, 174, 1047-1058.

209. Pastorino, F.; Brignole, C.; Di Paolo, D.; Nico, B.; Pezzolo, A.; Marimpietri, D.; Pagnan, G.; Piccardi, F.; Cilli, M.; Longhi, R.; Ribatti, D.; Corti, A.; Allen, T.M.; Ponzoni, M. Targeting liposomal chemotherapy via both tumor cell-specific and tumor-vasculature-specific ligands potentiates therapeutic efficacy. Cancer Res. 2006, 66, 10073-10082.

210. Powell, J.A.; Mohamed, S.N.; Kerr, J.S.; Mousa, S.A. Antiangiogenesis efficacy of nitric oxide donors. J. Cell. Biochem. 2000, 80, 104-114.

211. Chen, N.T.; Corey, E.J.; Folkman, J. Potentation of angiostatic steroids by a synthetic inhibitor of arylsulfatase. Lab. Invest. 1988, 59, 493-499.

212. Dings, R.P.; Chen, X.; Hellebrekers, D.M.; van Eijk, L.I.; Zhang, Y.; Hoye, T.R.; Griffioen, A.W.; Mayo, K.H. Design of nonpeptidic topomimetics of antiangiogenic proteins with antitumor activities. J. Natl. Cancer Inst. 2006, 98, 932-936. 
213. Leong, K.G.; Hu, X.; Li, L.; Noseda, M.; Larrivée, B.; Hull, C.; Hood, L.; Wong, F.; Karsan, A. Activated Notch4 inhibits angiogenesis: Role of beta 1-integrin activation. Mol. Cell. Biol. 2002, 22, 2830-2841.

214. Destouches, D.; El Khoury, D.; Hamma-Kourbali, Y.; Krust, B.; Albanese, P.; Katsoris, P.; Guichard, G.; Briand, J.P.; Courty, J.; Hovanessian, A.G. Suppression of tumor growth and angiogenesis by a specific antagonist of the cell-surface expressed nucleolin. PLOS ONE 2008, 3, e2518.

215. Marcinkiewicz, C.; Weinreb, P.H.; Calvete, J.J.; Kisiel, D.G.; Mousa, S.A.; Tuszynski, G.P.; Lobb, R.R. Obtustatin: A potent selective inhibitor of alphalbetal integrin in vitro and angiogenesis in vivo. Cancer Res. 2003, 63, 2020-2023.

216. Thippeswamy, G.; Sheela, M.L.; Salimath, B.P. Octacosanol isolated from Tinospora cordifolia downregulates VEGF gene expression by inhibiting nuclear translocation of NF- $<$ kappa $>\mathrm{B}$ and its DNA binding activity. Eur. J. Pharmacol. 2008, 588, 141-150.

217. Shahan, T.; Grant, D.; Tootell, M.; Ziaie, Z.; Ohno, N.; Mousa, S.; Mohamad, S.; Delisser, H.; Kefalides, N. Oncothanin, a peptide from the alpha3 chain of type IV collagen, modifies endothelial cell function and inhibits angiogenesis. Connect. Tissue Res. 2004, 45, 151-163.

218. Liekens, S.; Bronckaers, A.; Hernández, A.I.; Priego, E.M.; Casanova, E.; Camarasa, M.J.; Pérez-Pérez, M.J.; Balzarini, J. 5'-O-tritylated nucleoside derivatives: Inhibition of thymidine phosphorylase and angiogenesis. Mol. Pharmacol. 2006, 70, 501-509.

219. Dai, X.; Cui, S.G.; Wang, T.; Liu, Q.; Song, H.J.; Wang, R. Endogenous opioid peptides, endomorphin-1 and deltorphin I, stimulate angiogenesis in the CAM assay. Eur. J. Pharmacol. 2008, 579, 269-275.

220. Lee, H.J.; Lee, E.O.; Rhee, Y.H.; Ahn KS; Li, G.X.; Jiang, C.; Lü, J.; Kim, S.H. An oriental herbal cocktail, ka-mi-kae-kyuk-tang, exerts anti-cancer activities by targeting angiogenesis, apoptosis and metastasis. Carcinogenesis 2006, 27, 2455-2463.

221. Qian, X.P.; Liu, BR.; Li M.; Hu J; Hu, W.J.; Zou, Z.Y.; Wang, L.F.; Yu, L.X. Inhibitory effect of oxaliplatin in combination with hyperthermia on angiogenesis. Zhonghua Zhong Liu Za Zhi 2007, 29, 826-829.

222. Stefansson, S.; Petitclere, E.; Wong, M.K.; McMahon, G.A.; Brooks, P.C.; Lawrence, D.A. Inhibition of angiogenesis in vivo by plasminogen activator inhibitor-1. J. Biol. Chem. 2001, 276, 8135-8141.

223. Tian, F.; Zhang, X.; Tong, Y.; Yi Y, Zhang, S.; Li, L.; Sun, P.; Lin, L.; Ding, J. PE, a new sulfated saponin from sea cucumber, exhibits anti-angiogenic and anti-tumor activities in vitro and in vivo. Cancer Biol. Ther. 2005, 4, 874-882.

224. Mu P ; Gao X ; Jia ZJ ; Zheng, R.L. Natural antioxidant pedicularioside G inhibits angiogenesis and tumourigenesis in vitro and in vivo. Basic. Clin. Pharmacol. Toxicol. 2008, 102, 30-34.

225. Huh, J.E.; Lee, E.O.; Kim, M.S.; Kang, K.S.; Kim, C.H.; Cha, B.C.; Surh, Y.J.; Kim, S.H. Penta-O-galloyl-beta-D-glucose suppresses tumor growth via inhibition of angiogenesis and stimulation of apoptosis: Roles of cyclooxygenase-2 and mitogen-activated protein kinase pathways. Carcinogenesis 2005, 26, 1436-1445. 
226. Rusnati M; Urbinati C; Caputo A; Possati, L.; Lortat-Jacob, H.; Giacca, M; Ribatti, D; Presta, M. Pentosan polysulfate as an inhibitor of extracellular HIV-1 Tat. J. Biol. Chem. 2001, 276, 22420-22425.

227. Rusnati, M.; Camozzi, M.; Moroni, E.; Bottazzi, B.; Peri, G.; Indraccolo, S.; Amadori, A.; Mantovani, A.; Presta, M. Selective recognition of fibroblast growth factor-2 by the long pentraxin PTX3 inhibits angiogenesis. Blood 2004, 104, 92-99.

228. Don, A.S.; Kisker, O.; Dilda, P.; Donoghue, N.; Zhao, X.; Decollogne, S.; Creighton, B.; Flynn, E.; Folkman, J.; Hogg, P.J. A peptide trivalent arsenical inhibits tumor angiogenesis by perturbing mitochondrial function in angiogenic endothelial cells. Cancer Cell 2003, 3, 497-509.

229. Loutrari, H.; Hatziapostolou, M.; Skouridou, V.; Papadimitriou, E.; Roussos, C.; Kolisis, F.N.; Papapetropoulos, A. Perillyl alcohol is an angiogenesis inhibitor. J. Pharmacol. Exp. Ther. 2004, 311, 568-575.

230. Aljada, A.; O'Connor, L.; Fu, Y.Y.; Mousa, S.A. PPAR gamma ligands, rosiglitazone and pioglitazone, inhibit bFGF- and VEGF-mediated angiogenesis. Angiogenesis 2008, 11, 361-367.

231. Brooks, P.C.; Silletti, S.; von Schalscha, T.; Friedlander, M.; Cheresh, D.A. Disruption of angiogenesis by PEX, a noncatalytic metalloproteinase fragment with integrin binding activity. Cell 1998, 92, 391-400.

232. Xiao, D.; Singh, S.V. Phenethyl isothiocyanate inhibits angiogenesis in vitro and ex vivo. Cancer Res. 2007, 67, 2239-2246.

233. Lim, E.J.; Kang, H.J.; Jung, H.J.; Park, E.H. Anti-angiogenic, anti-nociceptive activity of 4-hydroxybenzyl alcohol. J. Pharm. Pharmacol. 2007, 59, 1235-1240.

234. Schumacher, J.J.; Upadhyaya, P.; Ramaktishnan, S. Inhibition of vascular endothelial cells by 1,4-phenylenebis (methylene)selenocyanate--a novel chemopreventive organoselenium compound. Anticancer Res. 2001, 21, 1945-1951.

235. Tong, Y.; Zhang, X.; Tian, F.; Yi, Y.; Xu, Q.; Li, L.; Tong, L.; Lin, L.; Ding, J. Philinopside A, a novel marine-derived compound possessing dual anti-angiogenic and anti-tumor effects. Int. J. Cancer 2005, 114, 843-853.

236. Tsopanoglou, N.E.; Pipili-Synetos, E.; Maragoudakis, M.E. Thrombin promotes angiogenesis by a mechanism independent of fibrin formation. Am. J. Physiol. 1993, 264, C1302-C1307.

237. Gottfried, V.; Lindenboum, E.S.; Kimel, S. The chick chorionallantoic membrane (CAM) as an in vivo model for photodynamic therapy. J. Photochem. Photobiol. B. 1992, 12, 204-207.

238. Yi, E.Y.; Jeong, E.J.; Kang, D.W.; Joo, J.H.; Kwon, H.S.; Lee, S.H.; Park, S.K.; Chung, S.G.; Cho, E.H.; Kim, Y.J. Anti-angiogenic and anti-tumor apoptotic activities of a topoisomerase II inhibiting agent SJ-8026. Int. J. Oncol. 2005, 26, 1613-1620.

239. Kumar, C.S.; Chandru, H.; Sharada, A.C.; Thimmegowda, N.R.; Prasad, S.B.; Kumar, M.K., Rangappa, K.S. Synthesis and evaluation of 1-benzhydryl-sulfonyl-piperazine derivatives as inhibitors of tumor angiogenesis of mouse ehrlich ascites tumor in vivo. Med. Chem. 2008, 4 , 466-472.

240. Shapiro, R.; Vallee, B.L. Human placental ribonuclease inhibitor abolishes both angiogenic and ribonucleolytic activities of angiogenin. Proc. Natl. Acad. Sci. USA 1987, 84, 2238-2241. 
241. Jeon, J.W.; Song, H.S.; Moon, E.J.; Park, S.Y.; Son, M.J.; Jung, S.Y.; Kim, J.T.; Nam, D.H.; Choi-Miura, N.H.; Kim, K.W.; Kim, Y.J. Anti-angiogenic action of plasma hyaluronan binding protein in human umbilical vein endothelial cells. Int. J. Oncol. 2006, 29, 209-215.

242. Li, J.; Dong, X.; Xu, Z.; Jiang, X.; Jiang, H.; Krissansen, G.W.; Sun, X. Endostatin gene therapy enhances the efficacy of paclitaxel to suppress breast cancers and metastases in mice. J. Biomed. Sci. 2008, 15, 99-109.

243. Morioka, H.; Morii, T.; Vogel, T.; Hornicek, F.J.; Weissbach, L. Interaction of plasminogen-related protein B with endothelial and smooth muscle cells in vitro. Exp. Cell Res. 2003, 287, 166-177.

244. Maione, T.E.; Gray, G.S.; Petro, J.; Hunt, A.J.; Donner, A.L.; Bauer, S.I.; Carson, H.F.; Sharpe, R.J. Inhibition of angiogenesis by recombinant human platelet factor-4 and related peptides. Science 1990, 247, 77-79.

245. Li, X.; Jiang, L.; Wang, Y.; Xiao, Y.; Huang, Y.; Yao, Q.; Yang, Y.; Wu, X. Inhibition of angiogenesis by a novel small peptide consisting of the active fragments of platet factor-4 and vasostatin. Cancer Lett. 2007, 256, 29-32.

246. Clapp, C.; Martial, J.A.; Guzman, R.C.; Rentier-Delure, F.; Weiner, R.I. The 16-kilodaldon $\mathrm{N}$-terminal fragment of human prolactin is a potent inhibitor of angiogenesis. Endocrinology 1993, 133, 1292-1299.

247. Pyriochou, A.; Olah, G.; Deitch, E.A.; Szabó, C.; Papapetropoulos, A. Inhibition of angigenesis by the poly(ADP-ribose) polymerase inhibior PJ-34. Int. J. Mol. Med. 2008, 22, 113-118.

248. Pacini, S.; Gulisano, M.; Vannucchi, S.; Ruggiero, M. Poly-L-lysine/heparin stimulates angiogenesis in chick embryo chorioallantoic membrane. Biochem. Biophys. Res. Commun. 2002, 290, 820-823.

249. Toi, M.; Bando, H.; Ramaschandan, C.; Melnick SJ, Imai A, Fife RS, Carr RE, Oikawa T, Lansky EP. Preliminary studies on the anti-angiogenic potential of pomegranate fractions in vitro and in vivo. Angiogenesis 2003, 6, 121-128.

250. Pepper, M.S.; Hazel, S.J.; Hümpel, M.; Schleuning, W.D. 8-prenylnaringenin, a novel phytoestrogen, inhibits angiogenesis in vitro and in vivo. J. Cell Physiol. 2004, 199, 98-107.

251. Ingber, D.; Folkman, J. Inhibition of angiogenesis through modulation of collagen metabolism. Lab. Invest. 1988, 59, 44-51.

252. Taylor, S.; Folkman, J. Protamine is an inhibitor of angiogenesis. Nature 1982, 297, 307-312.

253. Zania, P.; Kritikou, S.; Flordellis, C.S.; Maragoudakis, M.E.; Tsopanoglou, N.E. Blockade of angiogenesis by small molecule antagonists to protease-activated receptor-1: Association with endothelial cell growth suppression and induction of apoptosis. J. Pharmacol. Exp. Ther. 2006, 318, 246-254.

254. Rhim, T.Y.; Park, C.S.; Kim, E.; Kim, S.S. Human prothrombin fragment 1 and 2 inhibit bFGF-induced BCE cell growth. Biochem. Biophys. Res. Commun. 1998, 252, 513-516.

255. Gangjee, A.; Zeng, Y.; Ihnat, M.; Warnke LA; Green, D.W.; Kisliuk, R.L.; Lin, F.T. Novel 5-substituted, 2,4-diaminofuro[2,3-d]pyrimidines as multireceptor tyrosinen kinase and dihydrofolate reductase inhibitors with antiangiogenic and antitumor activity. Bioorg. Med. Chem.

2005, 13, 5475-5491. 
256. Presta, M.; Rusnati, M.; Belleri, M.; Morbidelli, L.; Ziche, M.; Ribatti, D. Purine analogue 6-methylmercaptopurine riboside inhibits early and late phase of the angiogenic process. Cancer Res. 1999, 59, 2417-2424.

257. Liekens, S.; Hernandez, A.I.; Ribatti, D.; De Clercq, E.; Camarasa, M.J.; Pérez-Pérez, M.J.; Balzarini, J. The nucleoside derivative 5'-O-trytyl-inosine (KIN59) suppresses thymidine phosphorylase-triggered angiogenesis via a noncompetitive mechanism of action. J. Biol. Chem. 2004, 279, 29598-29605.

258. Jung, M.H.; Lee, S.H.; Ahn, E.M.; Lee, Y.M. Decursin and decursinol angelate inhibit VEGF-induced angiogenesis via suppression of the VEGFR-2 signaling pathway. Carcinogenesis 2009, 30, 655-661.

259. Melkonian, G.; Eckelhoefer, H.; Wu, M.; Wang, Y.; Tong, C.; Riveles, K.; Talbot, P. Growth and angiogenesis are inhibited in vivo in developing tissues by pyrazine and its derivatives. Toxicol. Sci. 2003, 75, 393-401.

260. Matsumoto, T.; Turesson, I.; Book, M.; Gerwins, P.; Claesson-Welsh, L. p38 MAP kinase negatively regulates endothelial cell survival, proliferation, and differentiation in FGF-2-stimulated angiogenesis. J. Cell. Biol. 2002, 156, 149-160.

261. Tan, W.F.; Lin, L.P.; Li, Mh.; Zhang, Y.X.; Tong, Y.G.; Xiao, D.; Ding, J. Quercetin, a dietary-derived flavonoid, possesses antiangiogenic potential. Eur. J. Pharmacol. 2003, 459, $255-262$.

262. Isaacs, J.T.; Pili, R.; Qian, D.Z.; Dalrymple, S.L.; Garrison, J.B.; Kyprianou, N.; Björk, A.; Olsson, A.; Leanderson, T. Identification of ABR-215050 as lead second generation quinoline-3-carboxamide anti-angiogenic agent for the treatment of prostate cancer. Prostate 2006, 66, 1768-1778.

263. Oikawa, T.; Ito, H.; Ashino, H.; Toi, M.; Tominaga, T.; Morita, I.; Murota, S. Radicilor, a microbial cell differentiation modulator, inhibits in vivo angiogenesis. Eur. J. Pharmacol. 1993, 241, 221-227.

264. Urbinati, C.; Mitola, S.; Tanghetti, E.; Kumar, C.; Waltenberger, J.; Ribatti, D.; Presta, M.; Rusnati, M. Integrin alphavbeta3 as a target for blocking HIV-1 Tat-induced cell activation in vitro and angiogenesis in vivo. Arterioscler. Thromb. Vasc. Biol. 2005, 25, 2315-2320.

265. Youn, M.R.; Park, M.H.; Choi, C.K.; Ahn, B.C.; Kim, H.Y.; Kang, S.S.; Hong, Y.K.; Joe, Y.A.; Kim, J.S.; You, W.K.; Lee, H.S.; Chung, S.I.; Chang, S.I. Direct binding of recombinant plasminogen kringle 1-3 to angiogenin inhibits angiogenin-induced angiogenesis in the chick embryo CAM. Biochem. Biophys. Res. Commun. 2006, 343, 917-923.

266. Kim, T.H.; Kim, E.; Yoon, D.; Kim, J.; Rhim, T.Y.; Kim, S.S. Recombinant human prothrombin kringles have potent anti-angiogenic activities and inhibit Lewis lung carcinoma tumor growth and metastases. Angiogenesis 2002, 5, 191-201.

267. Kim, H.K.; Lee, S.Y.; Oh, H.K.; Kang, B.H.; Ku, H.J.; Lee, Y.; Shin, J.Y.; Hong, Y.K.; Joe, Y.A. Inhibition of endothelial cell proliferation by the recombinant kringle domain of tissue-type plasminogen activator. Biochem. Biophys. Res. Commun. 2003, 304, 740-746.

268. Kim, KS.; Hong, Y.K.; Joe, Y.A.; Lee, Y.; Shin, J.Y.; Park, H.E.; Lee, I.H.; Lee, S.Y.; Kang, D.K.; Chang, S.I.; Chung, S.I. Anti-angiogenic activity of the recombinant kringle domain of urokinase and its specific entry into endothelial cells. J. Biol. Chem. 2003, 278, 11449-11456. 
269. Jia, X.; Bian, L. Two-step chromatographic method for the separation and purification of recombinant angiogenesis inhibitor Kringle 5. SE. PU. 2007, 25, 344-347.

270. Oikawa, T.; Hirotani, K.; Nakamura, O.; Shudo, K.; Hiragun, A.; Iwaguchi, T. A highly potent antiangiogenic activity of retinoids. Cancer Lett. 1989, 48, 157-162.

271. Yeh, C.H.; Peng, H.C.; Yang, R.S.; Huang, T.F. Rhodostomin, a snake venom disintegrin, inhibits angiogenesis elicited by basic fibroblast growth factor and suppresses tumor growth by a selective alpha(v)beta(3) blockade of endothelial cells. Mol. Pharmacol. 2001, 59, 1333-1342.

272. Michaelis, M.; Michaelis, R.; Suhan, T.; Schmidt, H.; Mohamed, A.; Doerr, H.W.; Cinatl, J.Jr. Ribavirin inhibits angiogenesis by tetrahydrobiopterin depletion. FASEB J. 2007, 21, 81-87.

273. Chatterjee, J.; Maiti, T.K.; Dasgupta, S. Isolation and partial characterization of ribonuclease inhibitor from goat liver. Protein Pept. Lett. 2006, 13, 779-783.

274. Panigrahy, D.; Singer, S.; Shen, L.Q.; Butterfield, C.E.; Freedman, D.A.; Chen, E.J.; Moses, M.A.; Kilroy, S.; Duensing, S.; Fletcher, C.; Fletcher, J.A.; Hlatky, L.; Hahnfeldt, P.; Folkman, J.; Kaipainen, A. PPARgamma ligands inhibit primary tumor growth and metastasis by inhibiting angiogenesis. J. Clin. Invest. 2002, 110, 923-932.

275. Vacca, A.; Bruno, M.; Boccarelli, A.; Coluccia, M.; Ribatti, D.; Bergamo, A.; Garbisa, S.; Sartor, L.; Sava, G. Inhibition of endothelial cell functions and of angiogenesis by the metastasis inhibitor NAMI-A. Br. J. Cancer 2002, 86, 993-998.

276. Zhao, J.; Miao, J.; Zhao, B.; Zhang, S.; Yin, D. Safrole oxide inhibits angiogenesis by inducing apoptosis. FEBS Lett. 2005, 43, 69-74.

277. Kang, I.C.; Lee, Y.D.; Kim, D.S. A novel disintegrin salmosin inhibits tumor angiogenesis. Cancer Res. 1999, 59, 3754-3760.

278. Komi, Y.; Ohno, O.; Suzuki, Y.; Shimamura, M.; Shimokado, K.; Umezawa, K.; Kojima, S. Inhibition of tumor angiogenesis by targeting endothelial surface ATP synthase with sangivamycin. Jpn. J. Clin. Oncol. 2007, 37, 867-873.

279. Eun, J.P.; Koh, G.Y. Suppression of angiogenesis by the plant alkaloid, sanguinarine. Biochem. Biophys. Res. Commun. 2004, 317, 618-624.

280. Yoo, H.J.; Kang, H.J.; Jung, H.J.; Kim, K.; Lim, C.J.; Park, E.H. Anti-inflammatory, antiantinociceptive and anti-inflammatory activities of Saururus chinensis extract. J. Ethnopharmacol. 2008, 120, 282-286.

281. Jung HJ; Kang HJ; Song YS; Park, E.H.; Kim, Y.M.; Lim, C.J. Anti-inflammatory , anti-angiogenic and anti-nociceptive activities of Sedum sarmentosum extract. $J$. Ethnopharmacol. 2008, 116, 138-143.

282. Richardson, M.; Liu, L.; Dunphy, L.; Wong, D.; Sun, Y.; Viswanathan, K.; Singh, G.; Lucas, A. Viral serpin, Serp-1, inhibits endogenous angiogenesis in the chicken chorioallantoic membrane model. Cardiovasc. Pathol. 2007, 16, 191-202.

283. Hussain, S.; Slevin, M.; Matou, S.; Ahmed, N.; Choudhary, M.I.; Ranjit, R.; West, D.; Gaffney, J. Anti-angiogenic activity of sesterterpenes; natural product inhibitors of FGF-2 induced angiogenesis. Angiogenesis 2008, 11, 245-256.

284. Hagedorn, M.; Zilberberg, L.; Wilting, J.; Canron, X.; Carrabba, G.; Giussani, C.; Pluderi, M.; Bello, L.; Bikfalvi, A. Domain swapping in a $\mathrm{COOH}$-terminal fragment of platelet factor 4 generates potent angiogenesis inhibitors. Cancer Res. 2002, 62, 6884-6890. 
285. Casu, B.; Guerrini, M.; Naggi, A.; Perez, M.; Torri, G.; Ribatti, D.; Carminati, P.; Giannini, G.; Penco, S.; Pisano, C.; Belleri, M.; Rusnati, M.; Presta, M. Short heparin sequences spaced by glycol-split uronate residues are antagonists of fibroblast growth factor 2 and angiogenesis inhibitors. Biochemistry 2002, 41, 10519-10528.

286. Tournaire, R.; Simon, M.P.; le Noble, F.; Eichmann, A.; England, P.; Pouysségur, J. A short synthetic peptide inhibits signal transduction, migration and angiogenesis mediated by Tie2 receptor. EMBO Rep. 2004, 5, 262-267.

287. Park, H.J.; Kong, D.; Iruela-Arispe, L.; Begley, U.; Tang, D.; Galper, J.B. 3-hydroxy-3-methylglutaryl coenzyme A reductase inhibitors interfere with angiogenesis by inhibiting the geranylgeranylation of RhoA. Circ. Res. 2002, 91, 143-150.

288. Yi, E.Y.; Jeong, E.J.; Song, H.S.; Lee, M.S.; Kang, D.W.; Joo, J.H.; Kwon, H.S.; Lee, S.H.; Park, S.K.; Chung, S.G.; Cho, E.H.; Kim, Y.J. Anti-angiogenic and anti-tumor apoptotic activities of SJ-8002, a new piperazine derivative. Int. J. Oncol. 2004, 25, 365-372.

289. Jia, L.; Wu, C.C.; Guo, W.; Young, X. Antiangiogenic effects of S-nitrosocaptopril crystals as a nitric oxide donor. Eur. J. Pharmacol. 2000, 391, 137-144.

290. Xu, F.; Song, D.; Zhen, Y. Inhibition of tumor metastasis by sodium caffeate and its effect on angiogenesis. Oncology 2004, 67, 88-92.

291. Xu, Y.; Pan, R.L.; Chang, Q.; Qin, M.; Liu, Y.; Tang, J.T. Experimental study of Solanum nigrum on inhibiting angiogenesis in chick chorioallantoic membrane. Zhongguo Zhong Yao Za Zhi 2008, 33, 549-552.

292. Woltering, E.A.; Barrie, R.; O’Dorisio, T.M.; Arce, D.; Ure, T.; Cramer, A.; Holmes, D.; Robertson, J.; Fassler, J. Somatostatin analogues inhibit angiogenesis in the chick chorioallantoic membrane. J. Surg. Res. 1991, 50, 245-251.

293. Wrasidlo, W.; Mielgo, A.; Torres, V.A.; Barbero, S.; Stoletov, K.; Suyama, T.L.; Klemke, R.L.; Gerwick, W.H.; Carson, D.A.; Stupack, D.G. The marine lipopeptide somocystinamide A triggers apoptosis via caspase 8. Proc. Natl. Acad. Sci. USA 2008, 105, 2313-2318.

294. Su, S.J.; Yeh, T.M.; Chuang, W.J.; Ho, C.L.; Chang, K.L.; Cheng, H.L.; Liu, H.S.; Cheng, H.L.; Hsu, P.Y.; Chow, N.H. The novel targets for anti-angiogenesis of genistein on human cancer cells. Biochem. Pharmacol. 2005, 69, 307-318.

295. Jackson, J.K.; Burt, H.M.; Oktaba, A.M.; Hunter, W.; Scheid, M.P.; Mouhajir, F.; Lauener, R.W.; Shen, Y.; Salari, H.; Duronio, V. The antineoplastic ether lipid, s-phosphonate, selectively induces apoptosis in human leukemic cells and exhibits antiangiogenic and apoptotic activity on the chorioallantoic membrane of the chick embryo. Cancer Chemother. Pharmacol. 1998, 41, 326-332.

296. Klauber, N.; Browne, F.; Anand-Apte, B.; D'Amato, R.J. New activity of spironolactone. Inhibition of angiogenesis in vitro and in vivo. Circulation 1996, 94, 2566-2571.

297. Sills, A.K.; Williams, J.I.; Tyler, B.M.; Epstein, D.S.; Sipos, E.P.; Davis, J.D.; McLane, M.P.; Pitchford, S.; Cheshire, K.; Gannon, F.H.; Kinney, W.A.; Chao, T.L.; Donowitz, M.; Laterra, J.; Zasloff, M.; Brem, H. Squalamine inhibits angiogenesis and solid tumor growth in vivo and perturbs embryonic vasculature. Cancer Res. 1998, 58, 2784-2792.

298. Oikawa, T.; Shimamura, M.; Ashino, H. Inhibition of angiogenesis by staurosporine, a potent protein kinase inhibitor. J. Antibiot. 1992, 45, 1155-1160. 
299. Leali, D.; Belleri, M.; Urbinati, C.; Coltrini, D.; Oreste, P.; Zoppetti, G.; Ribatti, D.; Rusnati, M; Presta M. Fibroblast growth factor-2 antagonist activity and angiostatic capacity of solfate Escherìchia coli K5 polysaccharide derivatives. J. Biol. Chem. 2001, 176, 37900-37908.

300. Jakobson, A.M.; Hahnenberger, R.; Magnusson, A. Antiangiogenic effect of heparin and other sulphated glycosamynoglicans in the chick embryo chorioallantoic membrane. Pharmac. Toxic. 1991, 69, 122-126.

301. Tanaka, N.G.; Sakampto, N.; Inoue, K.; Korenaga, H.; Kadoya, S.; Ogawa, H.; Osada, Y. Antitumor effects of an antiangiogenic polysaccharide from an Arthrobacter species with or without a steroid. Cancer Res. 1989, 49, 6727-6730.

302. Inoue, K.; Korenaga, H.; Tanaka, N.G. The sulfated polysaccharide peptidoglycan complex potently inhibits embryonic angiogenesis and tumor growth in the presence of cortisone acetate. Carboydr Res. 1988, 181, 135-142.

303. Sola, F.; Farao, M.; Pesenti, E.; Marsiglio, A.; Mongelli, N.; Grandi, M. Antitumor activity of FCE 26644 a new growth-factor complexing molecule. Cancer Chemother. Pharmacol. 1995, 36, 217-222.

304. Ciomei, M.; Pastori, W.; Mariani, M.; Sola, F.; Grandi M; Mongelli N. New sulfonated distamycin A derivatives with bFGF complexing activity. Biochem. Pharmacol. 1994, 47, 295-302.

305. Liekens S; Neyts J; Degrève B; De Clercq, E. The sulfonic acid polymers PAMPS [poly(2-acrylamido-2-methyl-1-propanesulfonic acid)] and related analogues are highly potent inhibitors of angiogenesis. Oncol. Res. 1997, 9, 173-181.

306. Morimoto-Tomita, M.; Uchimura, K.; Bistrup, A.; Lum, D.H.; Egeblad, M.; Boudreau, N.; Werb, Z.; Rosen, S.D. Sulf-2, a proangiogenic separa sulfate endosulfatase, is upregulated in breast cancer. Neoplasia 2005, 7, 1001-1010.

307. Pyriochou, A.; Tsigkos, S.; Vassilakopoulos, S.; Cottin, T.; Zhou, Z.; Gourzoulidou, E.; Roussos, C.; Waldmann, H.; Giannis, A.; Papapetropoulos A. Anti-angiogenic properties of a sulindac analogue. Br. J. Pharmacol. 2007, 152, 1207-1214.

308. Elwich-Fils, S.; Soltysiak-Pawluczuk, D.; Splawiński, J. Anti-angiogenic and apoptotic effects of metabolites of sulindac on chick embryo chorioallantoic membrane. Hybrid Hybridomics 2003, 22, 55-60.

309. Danesi, R.; Del Bianchi, S.; Soldani, P.; Campagni, A.; La Rocca, R.V.; Myers, C.E.; Paparelli, A.; Del Tacca, M. Suramin inhibits bFGF-induced endothelial cell proliferation and angiogenesis in the chick chorioallantoic membrane. Br. J. Cancer 1993, 68, 932-938.

310. Gagliardi, A.R.; Kassack, M.; Kreimeyer, A.; Muller, G.; Nickel, P.; Collins, D.C. Antiangiogenic and antiproliferative activity of suramin analogues. Cancer Chemother. Pharmacol. 1998, 41, $117-124$.

311. Wilks, J.W.; Scott, P.A.; Vrba LK; Cocuzza, J.M. Inhibition of angiogenesis with combination treatments of angiostatic steroids and suramin. Int. J. Radiol. 1991, 60, 73-77.

312. Soriano, J.V.; Liu, N.; Gao, Y.; Yao, Z.J.; Ishibashi, T.; Underhill, C.; Burke, T.R.; Bottaro, D.P. Inhibition of angiogenesis by growth factor receptor bound protein 2-Src homology 2 domain bound antagonists. Mol. Cancer Ther. 2004, 3, 1289-1299.

313. Mousa SA; Mohamed, S.; Wexler, E.J.; Kerr, J.S. Antiangiogenesis and anticancer efficacy of TA138, a novel alphavbeta3 antagonist. Anticancer Res. 2005, 25, 197-206. 
314. Jeon, H.J.; Kang, H.J.; Jung, H.J.; Kang, Y.S.; Lim, C.J.; Kim, Y.M.; Park, E.H. Anti-inflammatory activity of Taraxacum officinale. J. Ethnopharmacol. 2008, 115, 82-88.

315. Zhang, Y.; He, L.; Meng, L.; Luo, W.; Xu, X. Suppression of tumor-induced angiogenesis by taspine isolated from Radix et Rhizoma Leonticis and its mechanism of action in vitro. Cancer Lett. 2008, 262, 103-113.

316. Nozaki, Y.; Hida, T.; Iinuma, S.; Ishii, T.; Sudo, K.; Muroi, M.; Kanamaru, T. Tau-1120, a new anthracycline with potent angiostatic activity. J. Antibiot. 1993, 46, 569-579.

317. Dordunoo, S.K.; Jackson, J.K.; Arsenault, L.A.; Oktaba, A.M.; Hunter, W.L.; Burt, H.M. Taxol encapsulation in poly(epsilon-caprolactone) microspheres. Cancer Chemother. Pharmacol. 1995, 36, 279-282.

318. Kurzen, H.; Schmitt, S.; Näher, H.; Möhler T. Inhibition of angiogenesis by non-toxic doses of temozolomide. Anticancer Drugs 2003, 14, 515-522.

319. Saito, Y.; Shiota, Y.; Nishisaka, M.; Owaki, T.; Shimamura, M.; Fukai, F. Inhibition of angiogenesis by a tenascin-c peptide which is capable of activating beta1-integrins. Biol. Pharm. Bull. 2008, 31, 1003-1007.

320. Ho, P.Y.; Liang, Y.C.; Ho, Y.S.; Chen, C.T.; Lee, W.S. Inhibition of human vascular endothelial cells proliferation by terbinafine. Int. J. Cancer 2004, 111, 51-59.

321. Liu, M.Z.; Tang, J.Z.; Zhang, J.S.; Chou, Q.; Huang, Y.Y. Angiogenesis inhibition in vascular endothelial cells by terpenoid compounds from Bletilla striata is via apoptosis pathway. Fen $\mathrm{Zi} \mathrm{Xi}$ Bao Sheng Wu Xue Bao 2008, 41, 383-392.

322. Mousa, S.A.; Bergh, J.J.; Dier, E.; Rebbaa, A.; O'Connor, L.J.; Yalcin, M.; Aljada, A.; Dyskin, E.; Davis, F.B.; Lin, H.Y.; Davis, P.J. Tetraiodothyroacetic acid, a small molecule integrin ligand, blocks angiogenesis induced by vascular endothelial growth factor and basic fibroblast growth factor. Angiogenesis 2008, 11, 183-190.

323. Ponticelli, S.; Marasco, D.; Tarallo, V.; Albuquerque, R.J.; Mitola, S.; Takeda, A.; Stassen, J.M.; Presta, M.; Ambati, J.; Ruvo, M.; De Falco, S. Modulation of angiogenesis by a tetrameric tripeptide that antagonizes vascular endothelial growth factor receptor 1. J. Biol. Chem. 2008, 283, 34250-34259.

324. Parson-Wingerter, P.; Elliott, K.E.; Farr, A.G.; Radhakrishnan, K.; Clark, J.I.; Sage, E.H. Generational analysis reveals that TGF-beta 1 inhibits the rate of angiogenesis in vivo by selective decrease in the number of new vessels. Microvasc. Res. 2000, 59, 221-232.

325. Presta M; Belleri M; Vacca A; Ribatti D. Anti-angiogenic activity of the purine analog 6-thioguanine. Leukemia 2002, 16, 1490-1499.

326. Chandrasekaran, L.; He, C.Z.; Al-Barazi, H.; Krutzsch, H.C.; Iruela-Arispe, M.L.; Roberts, D.D. Cell contact-dependent activation of alpha3beta1 integrin modulates endothelial cell responses to thrombospondin-1. Mol. Biol. Cell. 2000, 11, 2885-2900.

327. Margosio, B.; Rusnati, M.; Bonezzi, K.; Cordes BL; Annis, D.S.; Urbinati, C.; Giavazzi, R.; Presta, M.; Ribatti, D.; Mosher, D.F.; Taraboletti, G. Fibroblast growth factor-2 binding to the thrombospondin-1 type 111 repeats, a novel antiangiogenic domain. Int. J. Biochem. Cell Biol. 2008, 40, 700-709. 
328. Koutrafouri, V.; Leondiadis, L.; Avgoustakis, K.; Livaniou, E.; Czarnecki, J.; Ithakissios, D.S.; Evangelatos, G.P. Effect of thymosin peptides on the chick chorioallantoic membrane angiogenesis model. Biochim. Biophys. Acta 2001, 1568, 60-66.

329. Mousa, S.A.; Mohamed, S. Anti-angiogenic mechanisms and efficacy of the low molecular weight heparin, tinzaparin: Anti-cancer efficacy. Oncol. Rep. 2004, 12, 683-688.

330. Anand-Apte, B.; Pepper, M.S.; Yost, E.; Montesano, R.; Olsen, B.; Murphy, G.; Apte, S.S.; Zetter, B. Inhibition of angiogenesis by tissue inhibitor of metalloproteinase-3. Invest. Ophthalmol. Vis. Sci. 1997, 38, 817-823.

331. Hembrough, T.A.; Ruiz, J.F.; Swerdlow, B.M.; Swartz, G.M.; Hammers, H.J.; Zhang, L.; Plum, S.M.; Williams, M.S.; Strickland, D.K.; Pribluda, V.S. Identification and characterization of a very low density lipoprotein receptor-binding peptide from tissue factor pathway inhibitor that has antitumor and antiangiogenic activity. Blood 2004, 103, 3374-3380.

332. Bastaki, M.; Missirlis, E.; Klouras, N.; Karakiulakis, G.; Maragoudakis, M.E. Suppression of angiogenesis by the antitumor agent titanocene dichloride. Eur. J. Pharmacol. 1994, 251, 263-269.

333. Minischetti, M.; Vacca, A.; Ribatti, D.; Iurlaro, M.; Ria, R.; Pellegrino, A.; Gasparini, G.; Dammacco, A.F. TNP-470 and recombinant human interferon- $\alpha 2 \mathrm{a}$ inhibit angiogenesis synergistically. Br. J. Haematol. 2000, 109, 829-837.

334. Nakagawa, K.; Shibata, A.; Yamashita, S.; Tsuzuki, T.; Kariya, J.; Oikawa, S.; Miyazawa, T. In vivo angiogenesis is suppressed by unsaturated vitamin E, tocotrienol. J. Nutr. 2007, 137, 1938-1943.

335. Miyazawa, T.; Tsuzuki, T.; Nakagawa, K.; Igarashi, M. Antiangiogenic potency of vitamin E. Ann. N.Y. Acad. Sci. 2004, 1031, 401-404.

336. Jackson, J.K.; Higo, T.; Hunter, W.L.; Burt, H.M. Topoisomerase inhibitors as anti-arthritic agents. Inflamm. Res. 2008, 57, 126-134.

337. Yang X, Luo P, Yang B; He, Q. Antiangiogenesis response of endothelial cells to the antitumor drug 10-methoxy-9-nitrocamptothecin. Pharmacol. Res. 2006, 54, 334-340.

338. Puppo, M.; Battaglia, F.; Ottaviano, C.; Delfino, S.; Ribatti, D.; Varesio, L.; Bosco, M.C. Topotecan inhibits vascular endothelial growth factor production and angigenic activity induced by hypoxia in human neuroblastoma by targeting hypoxia-inducible factor-1alpha and -2alpha. Mol. Cancer Ther. 2008, 7, 1974-1984.

339. Kim, M.S.; Lee, Y.M.; Moon, E.J.; Kim, S.E.; Lee, J.J.; Kim, K.W. Anti-angiogenic activity of torilin, a sesquiterpene compound isolated from Torilis japonica. Int. J. Cancer 2000, 87, 269-275.

340. Benelli, U.; Lepri, A.; Nardi, M.; Danesi, R.; Del Tacca, M. Trapidil inhibits endothelial cell proliferation and angiogenesis in the chick chorioallantoic membrane and in the rat cornea. $J$. Ocul. Pharmacol. Ther. 1995, 11, 157-166.

341. McKay, T.L.; Gedeon, D.J.; Vickerman, M.B.; Hylton, A.G.; Ribita, D.; Olar, H.H.; Kaiser, P.K.; Parsons-Wingerter, P. Selective inhibition of angiogenesis in small blood vessels and decrease in vessel diameter throughout the vascular tree by triamcinolone acetonide. Invest. Ophthalmol. Vis. Sci. 2008, 49, 1184-1190. 
342. Zhu, G.F.; Zhang, H.Q.; Hou, A.J.; Yang, Y.L.; Li, Q.S.; Zhang, C.C. Effects of three compounds extracted from Tripterygium wilfordii Hook on angiogenesis in chick chorioallantoic membrane. Zhong Xi Yi Jie Xue Bao 2007, 5, 517-520.

343. Ding, Y.; Zhang, J.; Hou, L.; Zhang, J.; Wang, Q.; Wang, S. Effects of triptolide on anti-angiogenesis by reducing expression of urokinase plasminogen activator. Sheng $\mathrm{Wu} \mathrm{Yi} \mathrm{Xue}$ Gong Cheng Xue Za Zhi 2005, 22, 778-781.

344. Sohn, K.H.; Lee, H.Y.; Chung, H.Y.; Young, H.S.; Yi, S.Y.; Kim, K.W. Anti-angiogenic activity of triterpene acids. Cancer Lett. 1995, 94, 213-218.

345. Molina, M.C.; Ferreira, V.; Valck, C.; Aguilar, L.; Orellana, J.; Rojas, A.; Ramirez, G.; Billetta, R.; Schwaeble, W.; Lemus, D.; Ferreira, A. An in vivo role for Trypanosoma cruzi calreticulin in antiangiogenesis. Mol. Biochem. Parasitol. 2005, 140, 133-140.

346. Mannell, H.; Hellwig, N.; Gloe, T.; Plank, C.; Sohn, H.Y.; Groesser, L.; Walzog, B.; Pohl, U.; Krotz, F. Inhibition of the tyrosine phosphatase SHP-2 suppresses angiogenesis in vitro and in vivo. J. Vasc. Res. 2008, 45, 153-163.

347. Watanabe, J.; Endo, Y.; Shimada, N.; Natsume, T.; Sasaki, T.; Kobayashi, M. Antiangiogenic activity of TZT-1027 (soblidotin) on chick chorioallantoic membrane and human umbilical vein endothelial cells. In Vivo 2007, 21, 297-304.

348. Jung, H.J.; Leon, H.J.; Lim, E.J.; Ahn, E.K.; Song, Y.S.; Lee, S.; Shin, K.H.; Lim, C.J.; Park, E.H. Anti-angiogenic activity of the methanol extract and its fractions of Ulmus davidiana var. japonica. J. Ethnopharmacol. 2007, 112, 406-409.

349. Pisano, C.; Aulicino, C.; Vesci, L.; Casu, B.; Naggi, A.; Torri, G.; Ribatti, D.; Belleri, M.; Rusnati, M.; Presta, M. Undersulfated, low-molecular-weight glycol-split heparin as an antiangiogenic VEGF antagonist. Glycobiology 2005, 15, 1-6.

350. Suh, H.; Jung, E.J.; Kim, T.H.; Lee, H.Y.; Park, Y.H.; Kim, K.W. Anti-angiogenic activity of ursodeoxycholic acid and its derivatives. Cancer Lett. 1997, 113, 117-122.

351. Michaelis, M.; Michaelis, U.R.; Fleming, I.; Suhan, T.; Cinatl, J.; Blaheta, R.A.; Hoffmann, K.; Kotchetkov, R.; Busse, R.; Nau, H.; Cinatl, J.Jr. Valproic acid inhibits angiogenesis in vitro and in vivo. Mol. Pharmacol. 2004, 65, 520-527.

352. Jung, H.J.; Song, Y.S.; Lim, C.J.; Park, E.H. Anti-angiogenic, anti-inflammatory and anti-nociceptive activities of vanillyl alcohol. Arch. Pharm. Res. 2008, 31, 1275-1279.

353. Zhai Y, Yu J, Irucla-Arispe ML; Huang, W.Q.; Wang, Z.; Hayes, A.J.; Lu, J.; Jiang, G.; Rajas, L.; Lippman, M.E.; Ni, J.; Yu, G.L.; Li, LY.. Inhibition of angiogenesis and breast cancer xenograft tumor graft growth by VEGI, a novel cytokine of the TNF superfamily. Int. J. Cancer 1999, 82, 131-136.

354. Kern, J.; Bauer, M.; Rychli, K.; Wojta, J.; Ritsch, A.; Gastl, G.; Gunsilius, E.; Untergasser, G. Alternative splicing of vasohibin-1 generates an inhibitor of endothelial cell proliferation, migration, and capillary tube formation. Arterioscler. Thromb. Vasc. Biol. 2008, 28, 478-484.

355. Ma, L.; Luo, L.; Qiao, H.; Dong, X.; Pan, S.; Jiang, H.; Krissansen, G.W.; Sun, X. Complete eradication of hepatocellular carcinomas by combined vasostatin gene therapy and B7H3-mediated immunotherapy. J. Hepatol. 2007, 46, 98-106. 
356. Ramakrishnan, S.; Olson, T.A.; Bautch, V.L.; Mohanraj, D. Vascular endothelial growth factor-toxin conjugate specifically inhibits KDR/FLK-1-positive endothelial cell proliferation in vitro and angiogenesis in vivo. Cancer Res. 1996, 56, 1324-1330.

357. Vacca, A.; Iurlaro, M.; Ribatti, D.; Minischetti, M.; Nico, B.; Ria, R.; Pellegrino, A.; Dammacco, F. Antiangiogenesis is produced by nontoxic doses of vinblastine. Blood 1999, 94, 4143-4155.

358. Marimpietri, D.; Nico, B.; Vacca, A.; Mangieri, D.; Catarsi, P.; Ponzoni, M.; Ribatti, D. Synergistic inhibition of human neuroblastoma-related angiogenesis by vinblastine and rapamycin. Oncogene 2005, 24, 6785-6795.

359. Kisker, O.; Onizuka, S.; Becker, C.M.; Fannon, M.; Flynn, E.; D'Amato, R.; Zetter, B.; Folkman, J.; Ray, R.; Swamy, N.; Pirie-Shepherd, S. Vitamin D binding protein-macrophage activating factor (DBP-maf) inhibits angiogenesis and tumor growth in mice. Neoplasia 2003, 5, 32-40.

360. Oikawa, T.; Hirotani, K.; Ogasawara, H.; Katayama, T.; Nakamura, O.; Iwaguchi, T.; Hiragun, A. Inhibition of angiogenesis by vitamin D3 analogues. Eur. J. Pharmacol. 1990, 178, 247-250.

361. Lutty, G.A.; Thompson, D.C.; Gallup, J.Y.; Mello, R.J.; Patz, A.; Fenselau, A. Vitreous: An inhibitor of retinal extract-induced neovascularization. Invest. Ophthalmol. Vis. Sci. 1983, 24, 52-56.

362. Bae, M.K.; Jeong, J.W.; Kim, S.H.; Kim, S.Y.; Kang, H.J.; Kim, D.M.; Bae, S.K.; Yun, I.; Trentin, G.A.; Rozakis-Adcock, M.; Kim, K.W. Tid-1 interacts with the von Hippel-Lindau protein and modulates angiogenesis by destabilization of HIF-1alpha. Cancer Res. 2005, 65, 2520-2525.

363. Lin, C.M.; Chang, H.; Chen, Y.H.; Wu, I.H.; Chiu, J.H. Wogonin inhibits IL-6-induced angiogenesis via down-regulation of VEGF and VEGFR-1, not VEGFR-2. Planta MED. 2006, 72, $1305-1310$.

364. Scavelli, C.; Di Pietro, G.; Cirulli, T.; Coluccia, M.; Boccarelli, A.; Giannini, T.; Mangialardi, G.; Bertieri, R.; Coluccia, A.M.; Ribatti, D.; Dammacco, F.; Vacca, A. Zoledronic acid affects over-angiogenesis phenotype of endothelial cells in patients with multiple myeloma. Mol. Cancer Ther. 2007, 6, 3256-3262.

(C) 2010 by the authors; license Molecular Diversity Preservation International, Basel, Switzerland. This article is an open-access article distributed under the terms and conditions of the Creative Commons Attribution license (http://creativecommons.org/licenses/by/3.0/). 